\title{
Contributions of Different Cloud Types to Feedbacks and Rapid Adjustments in CMIP5*
}

\author{
Mark D. Zelinka, Stephen A. Klein, And Karl E. TAYlor \\ Program for Climate Model Diagnosis and Intercomparison, Lawrence Livermore National Laboratory, Livermore, California \\ TIMOTHY ANDREWS AND MARK J. WEBB \\ Met Office Hadley Center, Exeter, United Kingdom \\ JONATHAN M. GREGORY \\ National Centre for Atmospheric Science, University of Reading, Reading, and Met Office Hadley Centre, Exeter, United Kingdom \\ PIERS M. FORSTER \\ University of Leeds, Leeds, United Kingdom
}

(Manuscript received 30 July 2012, in final form 9 January 2013)

\begin{abstract}
Using five climate model simulations of the response to an abrupt quadrupling of $\mathrm{CO}_{2}$, the authors perform the first simultaneous model intercomparison of cloud feedbacks and rapid radiative adjustments with cloud masking effects removed, partitioned among changes in cloud types and gross cloud properties. Upon $\mathrm{CO}_{2}$ quadrupling, clouds exhibit a rapid reduction in fractional coverage, cloud-top pressure, and optical depth, with each contributing equally to a $1.1 \mathrm{~W} \mathrm{~m}^{-2}$ net cloud radiative adjustment, primarily from shortwave radiation. Rapid reductions in midlevel clouds and optically thick clouds are important in reducing planetary albedo in every model. As the planet warms, clouds become fewer, higher, and thicker, and global mean net cloud feedback is positive in all but one model and results primarily from increased trapping of longwave radiation. As was true for earlier models, high cloud changes are the largest contributor to intermodel spread in longwave and shortwave cloud feedbacks, but low cloud changes are the largest contributor to the mean and spread in net cloud feedback. The importance of the negative optical depth feedback relative to the amount feedback at high latitudes is even more marked than in earlier models. The authors show that the negative longwave cloud adjustment inferred in previous studies is primarily caused by a $1.3 \mathrm{~W} \mathrm{~m}^{-2}$ cloud masking of $\mathrm{CO}_{2}$ forcing. Properly accounting for cloud masking increases net cloud feedback by $0.3 \mathrm{~W} \mathrm{~m}^{-2} \mathrm{~K}^{-1}$, whereas accounting for rapid adjustments reduces by $0.14 \mathrm{~W} \mathrm{~m}^{-2} \mathrm{~K}^{-1}$ the ensemble mean net cloud feedback through a combination of smaller positive cloud amount and altitude feedbacks and larger negative optical depth feedbacks.
\end{abstract}

\section{Introduction}

Although 30 years have passed since the Charney report (Charney et al. 1979) first synthesized the state of

\footnotetext{
* Supplemental information related to this paper is available at the Journals Online website: http://dx.doi.org/10.1175/JCLID-12-00555.s1.

Corresponding author address: Mark D. Zelinka, Program for Climate Model Diagnosis and Intercomparison, Lawrence Livermore National Laboratory, 7000 East Avenue, L-103, Livermore, CA 94551. E-mail: zelinka1@llnl.gov
}

climate science and noted the prominent role of radiative feedbacks in driving uncertainty in projections of future climate change, the current generation of climate models continues to exhibit a wide range of radiative feedbacks and climate sensitivities (Andrews et al. 2012b). For a given increase in greenhouse gas concentration, the ensemble of models predicts a range of warming magnitudes that is directly proportional to the magnitude of the radiative feedbacks that operate as the planet warms, and diversity in cloud feedbacks is consistently identified as the dominant source of this wide range (Dufresne and Bony 2008; Soden and Held 2006). This is not surprising considering the tremendous leverage of clouds on both 
TABLE 1. Attributes of cloud feedbacks computed using two types of diagnostics and two methodologies.

\begin{tabular}{|c|c|c|}
\hline \multirow[b]{2}{*}{ Diagnostic } & \multicolumn{2}{|c|}{ Methodology } \\
\hline & $\begin{array}{l}\Delta R / \Delta T_{s}, \text { where } \Delta=\text { anomaly at } \\
\text { end of perturbed run }\end{array}$ & $\begin{array}{c}\text { Slope of } \Delta R \text { against } \\
\Delta T_{s}(\text { Gregory method })\end{array}$ \\
\hline CRE anomalies & $\begin{array}{l}\text { I: Affected by masking; neglects rapid } \\
\text { adjustments }\end{array}$ & $\begin{array}{l}\text { II: Affected by masking; accounts } \\
\text { for rapid adjustments }\end{array}$ \\
\hline $\begin{array}{l}\text { Kernel-derived cloud-induced } \\
\text { radiation anomalies }\end{array}$ & $\begin{array}{l}\text { III: Not affected by masking; neglects } \\
\text { rapid adjustments }\end{array}$ & $\begin{array}{l}\text { IV: Not affected by masking; accounts } \\
\text { for rapid adjustments }\end{array}$ \\
\hline
\end{tabular}

the longwave (LW) and shortwave (SW) budget of the planet and the fact that they are produced from subgridscale parameterization schemes rather than explicitly modeled in GCMs.

The importance of clouds as feedback mechanisms has been appreciated since at least the early 1970s with the pioneering studies of Schneider (1972), Schneider and Dickinson (1974), and Cess $(1974,1975)$ and continues to be an active area of research. In general, simulations in which the planet warms because of increased $\mathrm{CO}_{2}$ exhibit an overall decrease in cloud fraction, except at high latitudes and in some tropical areas that become more favorable for convection (Wetherald and Manabe 1988; Senior and Mitchell 1993; Colman et al. 2001; Meehl et al. 2007; Zelinka et al. 2012b). They also exhibit increased cloud-top altitude as the troposphere deepens (Zelinka and Hartmann 2010; Zelinka et al. 2012b) and an increase in high-latitude cloud optical depth resulting from increases in cloud water content and phase changes (Senior and Mitchell 1993; Tsushima et al. 2006; Zelinka et al. $2012 b)$. These gross features are quite common to most GCM simulations. However, subtle changes to cloud properties that vary in space and time lead to significant spatiotemporal variability in the magnitudes of large and oppositely signed cloud feedbacks, of which the global mean cloud feedback is the small residual. Disparate responses of marine boundary layer clouds in the subsidence regions of the tropics and subtropics are the leading source of intermodel spread in global mean cloud feedback (Bony and Dufresne 2005; Webb et al. 2013).

Recently it has become apparent that clouds also respond directly to the greenhouse gas perturbation in such a way as to modify the radiative budget of the planet independently of their surface temperaturemediated effects (Gregory and Webb 2008). Such rapid responses of clouds arise because $\mathrm{CO}_{2}$ perturbations have an immediate effect on the radiative cooling rate and temperature structure of the atmosphere, even before the global mean surface temperature can respond (i.e., on a time scale of less than 1 month; Dong et al. 2009; Cao et al. 2012). Unlike radiative feedbacks, of which cloud feedback is one among several relevant for modifying the top-of-atmosphere (TOA) energy balance as the planet warms, rapid adjustments to $\mathrm{CO}_{2}$ are almost entirely a result of cloud changes (Andrews and Forster 2008; Colman and McAvaney 2011). It has been repeatedly shown that rapid reductions in the coverage of low and midlevel clouds upon introduction of $\mathrm{CO}_{2}$ play the dominant role in causing positive radiative adjustments through the attendant reduction in planetary albedo (Andrews and Forster 2008; Colman and McAvaney 2011; Watanabe et al. 2012; Wyant et al. 2012; Kamae and Watanabe 2013). These cloud reductions have been attributed to decreases in relative humidity in association with $\mathrm{CO}_{2}$-induced temperature increases (Colman and McAvaney 2011; Kamae and Watanabe 2012). For a thorough review of cloud adjustments to $\mathrm{CO}_{2}$, see Andrews et al. (2012a).

As first pointed out in Gregory and Webb (2008), many of the radiation anomalies resulting from cloud changes that are commonly included as part of the cloud feedback actually occur because of rapid cloud adjustments and are better thought of as part of the forcing. Webb et al. (2013) found that the contribution of variations in cloud feedback to the intermodel spread in climate sensitivity is about 4 times as large as that resulting from rapid cloud adjustments, though the latter is not negligible. Properly distinguishing between and quantifying the radiative implications of rapid cloud changes induced by $\mathrm{CO}_{2}$ perturbations and cloud changes that evolve linearly with increasing global mean surface temperature (temperature-mediated cloud changes) is thus necessary for 1) disentangling the role of $\mathrm{CO}_{2}$ from that of global mean surface temperature in causing clouds to change within a given model and 2) properly attributing intermodel spread of climate sensitivity to forcing versus feedback.

There are two main issues that cause difficulty in interpreting results from previous studies. The first is methodological, and the second involves the choice of diagnostics. Most studies to date (excluding those listed in the previous two paragraphs) have computed cloud feedbacks by simply taking some measure of the TOA radiative flux anomaly due to clouds between a perturbed and unperturbed climate and dividing by the global mean surface temperature change that occurred 
between climate states (categories I and III in Table 1). However, rapid cloud changes that are not temperature dependent may make a substantial contribution to the TOA flux anomaly, and failing to account for them may result in an estimated feedback of the wrong magnitude and even sign (Andrews and Forster 2008).

On the other hand, most studies-especially those evaluating an ensemble of models - that have computed the rapid cloud adjustment and cloud feedback have done so using anomalies in cloud radiative effect (CRE; the clear-sky minus all-sky upwelling radiative flux at the TOA) as their diagnostic (e.g., Andrews et al. 2012b). These are represented by category II in Table 1 . As pointed out in Zhang et al. (1994) and Soden et al. (2004, 2008), anomalies in CRE include contributions from changes in noncloud variables in such a way as to negatively bias the derived cloud feedback. Additionally, the presence of clouds masks a portion of the radiative forcing due to $\mathrm{CO}_{2}$ independent of any cloud response to it. Studies that have used more sophisticated techniques that avoid cloud masking have been performed only within a given model (e.g., Colman and McAvaney 2011; Watanabe et al. 2012; Wyant et al. 2012), only for fixed sea surface temperature (SST) simulations with perturbed $\mathrm{CO}_{2}$ (e.g., Wyant et al. 2012), and/or only in slab ocean models (Andrews and Forster 2008).

Thus, there is a need to separately quantify both cloud feedbacks and rapid adjustments across an ensemble of recent fully coupled atmosphere-ocean coupled GCM (AOGCM) integrations using time-evolving TOA radiation anomalies due solely to changes in cloud properties, with no influence from changes in noncloud fields (category IV in Table 1). Additionally, our study is motivated by a desire to diagnose in detail the changes in cloud types and cloud properties that are associated with feedbacks and rapid adjustments and to quantify their impacts on TOA fluxes. Doing so shines light on the physical mechanisms responsible for the adjustments and feedbacks and more clearly identifies the changes to cloud types and properties for which models agree and disagree.

The cloud radiative kernel technique (Zelinka et al. 2012a) is exceptionally well suited to this problem. Because the radiation anomalies computed with the cloud radiative kernels are due to cloud changes alone, they provide estimates of the cloud feedback and rapid radiative adjustment with no influence from noncloud changes. Furthermore, because the cloud radiative kernels quantify the sensitivity of TOA fluxes to cloud fraction perturbations for 49 different cloud types, the technique provides a quantitative partitioning of the rapid cloud adjustments and cloud feedbacks among cloud types separated by altitude and optical depth and among changes in the overall amount, altitude, and optical depth of clouds.
We describe our data and methodology in section 2, compute global mean cloud feedbacks and rapid adjustments in section 3, detail their spatial patterns in section 4 , and quantify the implications of diagnostic and methodological choices for the derived feedbacks and rapid adjustments in section 5. Finally, we summarize results and highlight some remaining questions in section 6 .

\section{Data and methodology}

We make use of monthly diagnostics from a variety of simulations from fully coupled AOGCMs available in the phase 5 of the Coupled Model Intercomparison Project (CMIP5)/Cloud Feedback Model Intercomparison Project phase 2 (CFMIP2) archive (Table 2). Henceforth we use the CMIP5 experiment nomenclature of Taylor et al. (2012). We analyze output from abrupt 4 xCO2 runs, which are branched from preindustrial control (piControl) runs by instantaneously quadrupling the atmospheric $\mathrm{CO}_{2}$ concentration from its preindustrial level and holding it fixed. We also use output from sstClim and sstClim $4 \mathrm{xCO} 2$ runs, which are atmosphere-only simulations in which climatological SSTs and sea ice from the piControl run are imposed, but with atmospheric $\mathrm{CO}_{2}$ levels fixed at preindustrial and quadrupled levels, respectively. Finally, we use output from $1 \mathrm{pctCO} 2$ runs that are forced by a compounding $1 \% \mathrm{yr}^{-1}$ increase in $\mathrm{CO}_{2}$ from preindustrial levels, reaching quadrupled levels $140 \mathrm{yr}$ after branching from piControl.

Each model analyzed in this study implemented the International Satellite Cloud Climatology Project (ISCCP) simulator (Klein and Jakob 1999; Webb et al. 2001), which translates the modeled cloud fields into a distribution of cloud fraction as a joint function of seven cloudtop pressure (CTP) ranges and seven cloud optical depth $\tau$ ranges in an analogous manner to the observational ISCCP cloud products. The six models listed in Table 2 were chosen because they are the currently available (as of December 2012) models that performed the necessary experiments with a correctly implemented ISCCP simulator. To verify proper simulator implementation, we compared the model-produced total cloud fraction diagnostic clt with the cloud fractions from the ISCCP simulator summed over all cloud types $C_{\text {tot }}$. In the models that have successfully implemented the simulator, the global mean $C_{\text {tot }}$ minus clt bias is no larger than $1.9 \%$ absolute and the RMS difference is no larger than $4.4 \%$ absolute.

As called for in the CMIP5 protocol, ISCCP simulator output is available for the full $30-50$-yr duration of the sstClim and sstClim $4 x \mathrm{xCO} 2$ experiments, for the first $20 \mathrm{yr}$ and last $20 \mathrm{yr}$ of the 150-yr abrupt $4 \mathrm{xCO} 2 \mathrm{run}$, and 
TABLE 2. Global climate models used in this study. Relevant diagnostics for the CCSM4 were provided only for the sstClim and sstClim4xCO2 runs. The MPI-ESM-LR provided only one abrupt4xCO2 ensemble member to the archive and did not provide relevant diagnostics from the sstClim or sstClim4xCO2 runs. Two configurations of the HadGEM2 are used in this study: HadGEM2-A is the atmospheric only configuration, while HadGEM2-ES includes all components of the earth system.

\begin{tabular}{|c|c|c|c|}
\hline Abbreviation & Model expansion & Modeling center & Reference \\
\hline CanESM2 & $\begin{array}{l}\text { Canadian Earth System Model, } \\
\text { version } 2\end{array}$ & $\begin{array}{l}\text { Canadian Centre for Climate Modelling } \\
\text { and Analysis }\end{array}$ & von Salzen et al. 2013 \\
\hline CCSM4 & $\begin{array}{l}\text { Community Climate System Model, } \\
\text { version } 4\end{array}$ & National Center for Atmospheric Research & Gent et al. 2011 \\
\hline HadGEM2 & $\begin{array}{l}\text { Hadley Centre Global Environmental } \\
\text { Model }\end{array}$ & Met Office Hadley Centre & Collins et al. 2011 \\
\hline MIROC5 & $\begin{array}{l}\text { Model for Interdisciplinary Research } \\
\text { on Climate } 5\end{array}$ & $\begin{array}{l}\text { Atmosphere and Ocean Research Institute } \\
\text { (University of Tokyo), National Institute for } \\
\text { Environmental Studies, and Japan Agency for } \\
\text { Marine-Earth Science and Technology }\end{array}$ & Watanabe et al. 2010 \\
\hline MPI-ESM-LR & $\begin{array}{l}\text { Max Planck Institute Earth System } \\
\text { Model, low resolution }\end{array}$ & Max Planck Institute for Meteorology & Stevens et al. 2013 \\
\hline MRI-CGCM3 & $\begin{array}{l}\text { Meteorological Research Institute } \\
\text { Coupled General Circulation } \\
\text { Model, version } 3\end{array}$ & Meteorological Research Institute & Yukimoto et al. 2011 \\
\hline
\end{tabular}

for the last $20 \mathrm{yr}$ of the $140-\mathrm{yr} 1 \mathrm{pctCO} 2$ run. Monthly mean climatologies in the sstClim $4 \mathrm{xCO} 2$ and sstClim runs are differenced to compute anomalies in what we will refer to as the "fixed-SST experiment." To compute anomalies in the abrupt $4 \mathrm{xCO} 2$ and $1 \mathrm{pctCO} 2$ runs, we subtract from each of the available 20 -yr periods the monthly mean annual cycle from the corresponding 20-yr portion of the piControl run.

For each model (except MPI-ESM-LR), a 12-member ensemble of abrupt $4 \mathrm{xCO} 2$ simulations is analyzed, with each one having branched from piControl in a different month of the year (Taylor et al. 2012). The first ensemble member is run for the 150-yr duration of the abrupt $4 \mathrm{xCO} 2$ simulation, whereas the others are run only for the first $5 \mathrm{yr}$ following quadrupling. Because each ensemble member is perturbed starting in a different month, averaging across all 12 members for each month provides monthly resolution of the early years of the perturbed simulation while not being sensitive to the month in which the perturbation occurred (DoutriauxBoucher et al. 2009).

A key feature of the cloud distributions provided by the ISCCP simulator is that cloud fraction in each bin of the histogram is a "radiatively relevant" nonoverlapped cloud fraction (from a TOA perspective). Thus, it is possible to quantify the individual contributions of changes of each cloud type to changes in the TOA LW and SW fluxes. Zelinka et al. (2012a) used a radiative transfer model (Fu and Liou 1993) to compute sensitivities of TOA fluxes $R$ to absolute perturbations of cloud fraction $C_{p \tau}$ of $1 \%$ in each of the 49 bins of the ISCCP histogram, which they refer to as cloud radiative kernels $K_{p \tau}$,

$$
K_{p \tau} \equiv \frac{\partial R}{\partial C_{p \tau}} .
$$

The subscripts $p$ and $\tau$ indicate that the field is a function of cloud-top pressure and optical depth.

The cloud radiative kernels, when multiplied by changes in ISCCP simulator-diagnosed cloud fraction $\Delta C_{p \tau}$ between a perturbed and unperturbed climate and summed over all CTP and $\tau$ categories, produce an estimate of the cloud-induced anomaly in upwelling TOA radiative fluxes $\Delta R_{C}$,

$$
\Delta R_{C}=\sum_{p=1}^{P} \sum_{\tau=1}^{T}\left(K_{p \tau} \Delta C_{p \tau}\right),
$$

where both $P$ and $T$ equal 7. Zelinka et al. (2012a) showed that the cloud feedbacks computed using values of $\Delta R_{C}$ derived in this manner agreed very well, both in the global mean and on a point-by-point basis with the adjusted change in cloud forcing method of Soden et al. (2008) and Shell et al. (2008), though agreement was generally better in the SW.

Gregory et al. (2004) showed that TOA radiation anomalies evolve linearly with increasing global mean surface air temperature anomaly $\Delta T_{s}$ following a step change in radiative forcing, implying that the planetary energy budget can be expressed in a simple linear form,

$$
\Delta R=F+\alpha \Delta T_{s},
$$

where $\Delta R$ is the net downwelling TOA radiative flux anomaly relative to the initial equilibrium state, $F$ is the 
effective climate forcing [using the terminology of Forster and Taylor (2006)], and $\alpha$ is the net climate feedback (including the Planck response and therefore negative for a stable climate). Here, $F$ is determined as the $y$ intercept and $\alpha$ as the slope of the ordinary least squares linear regression line of $\Delta R$ on $\Delta T_{s}$.

Here, we use this same diagnostic approach but use cloud-induced downwelling radiative flux anomalies $\Delta R_{C}$ computed with the cloud radiative kernels [Eq. (2)] to derive the cloud feedback $\alpha_{C}$ and the instantaneous radiation perturbation resulting from rapid cloud adjustments $F_{C}$,

$$
\Delta R_{C}=F_{C}+\alpha_{C} \Delta T_{s} .
$$

As will be shown below, global and annual mean values of $\Delta R_{C}$ vary linearly with global mean $\Delta T_{s}$, implying that $\alpha_{C}$ and $F_{C}$ are constants that can be estimated by linear regression of $\Delta R_{C}$ on $\Delta T_{s}$. The $y$ intercept of the regression line is an estimate of the cloud-induced radiative perturbation immediately after $\mathrm{CO}_{2}$ is quadrupled were the global mean $T_{s}$ to remain fixed, and we will refer to this as the rapid cloud radiative adjustment $F_{C}$. The slope of the regression line passing through the data points represents the change in cloud-induced radiative fluxes per Kelvin increase in global mean surface temperature, the cloud feedback $\alpha_{C}$. A positive slope $\left(\alpha_{C}>0\right)$ implies a positive cloud feedback, and vice versa. Uncertainties in regression slopes are estimated as the two standard deviation (i.e., $\sigma$ ) range of possible regression slopes computed from 1000 bootstrapped samples with replacement.

\section{Global mean rapid cloud adjustments and cloud feedbacks}

In Fig. 1, we show Gregory plots for cloud-related anomalies in LW (left column) and SW (right column) TOA radiative fluxes for the five models with the necessary diagnostics. Cloud-induced TOA radiative flux anomalies derived using cloud radiative kernels are shown in black and CRE anomalies are shown in gray. Regression lines are computed using the annual and global mean anomalies; the monthly resolved anomalies from the early portion of the run are not used in the regression so as to avoid giving the early anomalies undue influence on the slope. Figures 2 and 3 contain global mean values of $\mathrm{LW}, \mathrm{SW}$, and net rapid cloud adjustments and cloud feedbacks, respectively, for the five models analyzed. All global mean values and their uncertainties are provided in the supplementary tables.

Global mean values of $\triangle \mathrm{LWCRE}$ and cloud-induced LW flux anomalies behave remarkably linearly when plotted against the $\Delta T_{s}$ anomalies and rarely deviate from the regression lines. In all but one model (MRICGCM3), the kernel-derived LW cloud feedback is positive, though in both the MRI-CGCM3 and MIROC5 models it is statistically indistinguishable from zero. Even among this relatively small ensemble of five models, the LW cloud feedback spans a considerable range, from just under 0 to $0.8 \mathrm{~W} \mathrm{~m}^{-2} \mathrm{~K}^{-1}$. The kernel-derived LW cloud adjustment ( $y$ intercept) is indistinguishable from zero in all but the HadGEM2-ES and MRI-CGCM3 models, and in all models the LW impact of cloud adjustments is dwarfed by the direct impact of quadrupling $\mathrm{CO}_{2}$. Note that, in HadGEM2-ES, the early anomalies clearly deviate from the regression line, so even this model likely has a small negative LW cloud adjustment. These small and generally negative values of LW cloud adjustment are consistent with those listed in Table 1 of Andrews and Forster (2008).

The $\triangle$ SWCRE and cloud-induced SW flux anomalies also behave quite linearly when plotted against the $\Delta T_{s}$ anomalies, though, compared with the LW, larger deviations from the regression lines are apparent in the first few years after quadrupling, especially in the HadGEM2-ES and MRI-CGCM3 models (Fig. 1, right). The SW cloud feedbacks vary considerably among the models, though, unlike the LW cloud feedbacks, large magnitudes of either sign are possible, ranging from -0.3 to $0.3 \mathrm{~W} \mathrm{~m}^{-2} \mathrm{~K}^{-1}$. In contrast to the consistently small LW cloud adjustments, the SW cloud adjustments vary from -0.6 to $2.1 \mathrm{~W} \mathrm{~m}^{-2}$ and are always distinguishable from zero. The two models for which the intercept is negative (HadGEM2-ES and MRI-CGCM3) exhibit obvious deviations from the regression line in the first few years of the integration, and their early anomalies are positive. ${ }^{1}$ Clearly rapid cloud adjustments are much more relevant to the global mean SW budget than to the LW budget and-considering the early abrupt $4 \mathrm{xCO} 2$ anomalies and the fixed-SST experiment anomalies rather than the regression intercepts - tend to enhance the radiative forcing due to $\mathrm{CO}_{2}$, in accord with previous studies (Andrews and Forster 2008; Colman and McAvaney 2011; Watanabe et al. 2012; Webb et al. 2013; Wyant et al. 2012; Kamae and Watanabe 2012).

In Fig. 1, we show with red crosses the anomalies from the fixed-SST experiment, which give alternative estimates of the response of clouds to a $\mathrm{CO}_{2}$ quadrupling before appreciable surface warming occurs. (Note, however, that the land surface warms, which is reflected in a global mean warming of roughly $0.5 \mathrm{~K}$.) We have also

\footnotetext{
${ }^{1}$ For further discussion of the deviations from linearity in the early stages of the abrupt $4 \mathrm{xCO} 2$ simulation, please refer to section 4 of Andrews et al. (2012b).
} 

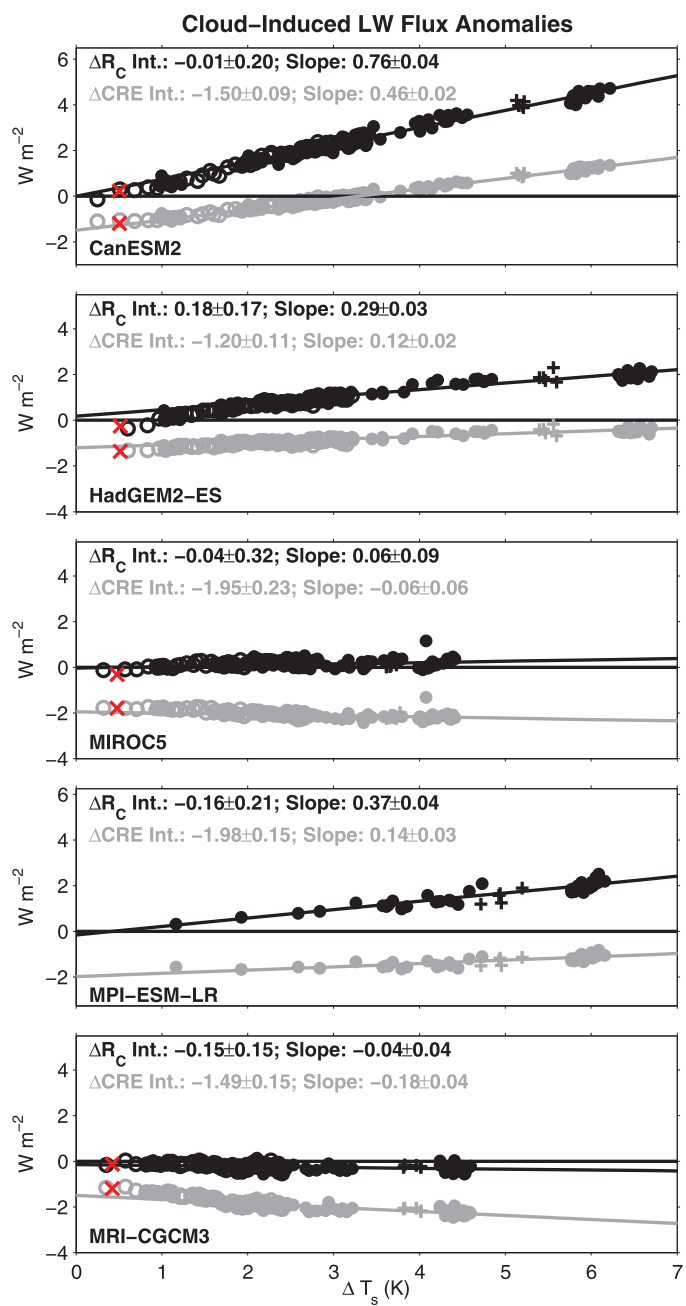
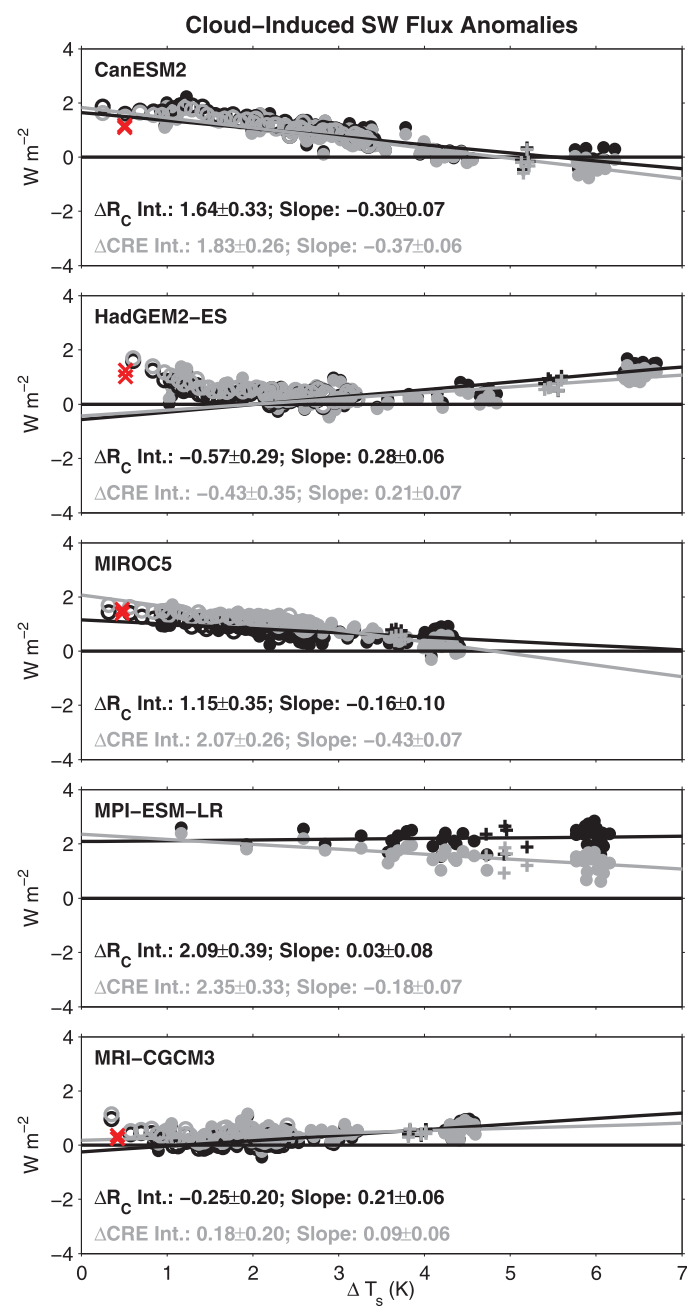

FIG. 1. Global mean anomalies in cloud-induced TOA (left) LW and (right) SW radiative fluxes derived using cloud radiative kernels (black) and CRE plotted against global mean $\Delta T_{s}$ (gray). Filled circles represent annual anomalies computed using the first ensemble member of the abrupt4xCO2 run. Unfilled circles represent the annually averaged monthly resolved anomalies computed using the 12-member ensembles available for the first $5 \mathrm{yr}$ of the abrupt4xCO2 run. Red crosses represent the anomalies derived from the fixed-SST experiment and plus signs represent the anomalies derived from the final $5 \mathrm{yr}$ of the $1 \mathrm{pctCO} 2$ runs. Lines represent the ordinary least squares regression of the annual and global mean abrupt4xCO2 anomalies on annual and global mean $\Delta T_{s}$, and the $y$ intercept and slope of these lines are displayed in each panel, along with their $2 \sigma$ uncertainties.

plotted plus signs in Fig. 1 that indicate the anomalies from the last $5 \mathrm{yr}$ of the $1 \mathrm{pctCO} 2$ runs (i.e., the $5 \mathrm{yr}$ closest to when $\mathrm{CO}_{2}$ concentrations are quadrupled from preindustrial). Both the $1 \mathrm{pctCO} 2$ and fixed-SST anomalies closely match the anomalies in the quadrupled $\mathrm{CO}_{2}$ runs, highlighting the robustness of these anomalies for a given increase in $\mathrm{CO}_{2}$ and surface temperature. That the $1 \mathrm{pctCO} 2$ anomalies lie along the regression line at the time in the $1 \mathrm{pctCO} 2$ run where the $\mathrm{CO}_{2}$ is close to 4 times its preindustrial value suggests that the cloud adjustments due solely to $\mathrm{CO}_{2}$ depend only on the prevailing $\mathrm{CO}_{2}$ concentration and not its history and that cloud feedbacks are the same in both experiments. This implies that the relative roles of $\mathrm{CO}_{2}$ and surface temperature anomalies in affecting clouds at any point in the $1 \mathrm{pctCO} 2$ runs may plausibly be inferred from the abrupt $4 \mathrm{xCO} 2$ experiments. Thus, information derived from highly idealized step-function forcing experiments are relevant to more realistic transient scenarios (e.g., Good et al. 2011, 2012).

\section{Spatial patterns of rapid cloud adjustments and temperature-mediated cloud anomalies}

In the following sections, we elucidate the threedimensional patterns of cloud anomalies that contribute 


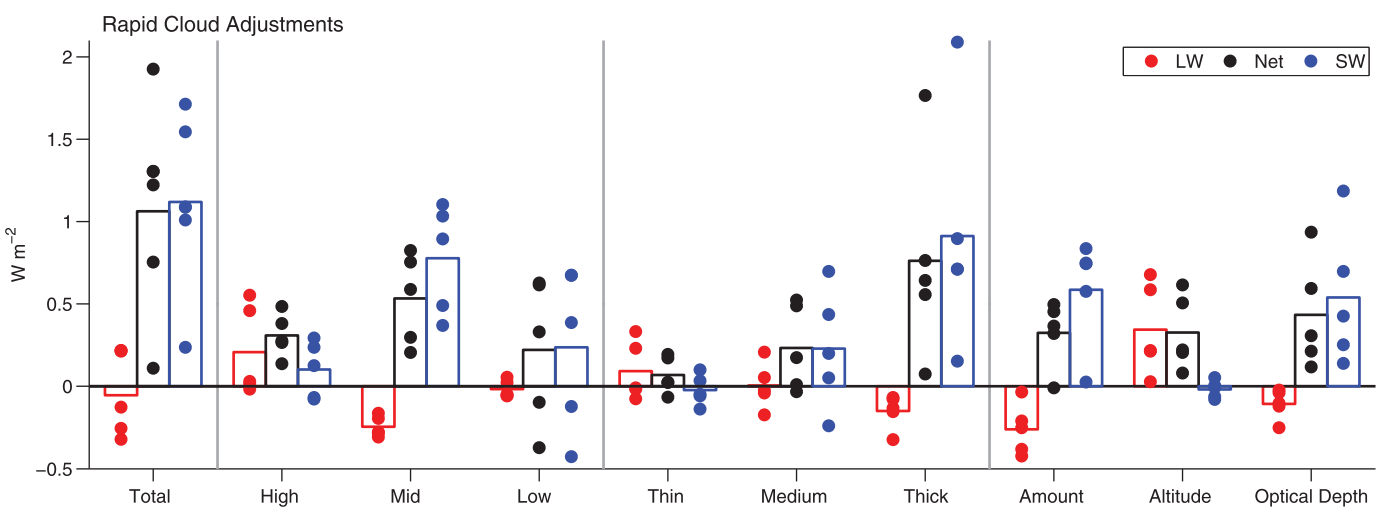

FIG. 2. Global mean cloud adjustments computed from the fixed-SST simulations, separated into components from the three major altitude, optical depth, and gross cloud property change categories. Each dot represents a single model, and the bars extend to the five-model mean. Vertical gray lines separate the groups of components whose sums equal the total rapid cloud radiative adjustment. Numerical values are provided in supplementary Table 1.

to the rapid cloud adjustments and cloud feedbacks. It is necessary to bear in mind that the cloud fractions provided by the ISCCP simulator represent only those cloud tops that are exposed to space and are therefore unobscured by overlying clouds. While this is a desirable feature for quantification of the radiative impact of changes in each cloud type, it makes interpretation of the true cloud changes more difficult, especially for lower clouds overlain by higher clouds. For example, at a location in which a reduction in low cloud fraction coincides with a larger reduction in overlying cloud fraction, the ISCCP simulator will "see" an increase in low cloud fraction because more of it became exposed to space. In addition, under certain circumstances the ISCCP simulator will misassign clouds to midlevels. This is done purposely to remain faithful to the ISCCP cloud retrieval process, which erroneously assigns clouds to midlevels when optically thin high clouds are present above low clouds (Marchand et al. 2010; Mace et al. 2011) or when low clouds are present under temperature inversions (Garay et al. 2008).

In the following sections, we identify any location in which changes in ISCCP-simulator-derived cloudiness are "real" as opposed to where they result from changes in obscuration. This is done using model-produced cloud amounts as detailed in appendix A.

\section{a. Spatial patterns of rapid cloud adjustments}

Hereafter, we calculate rapid adjustments using cloud anomalies from the fixed-SST experiment rather than as $y$ intercepts from the abrupt $4 \mathrm{xCO} 2$ run. Averaging over the 30-yr fixed-SST experiment reduces the sensitivity of

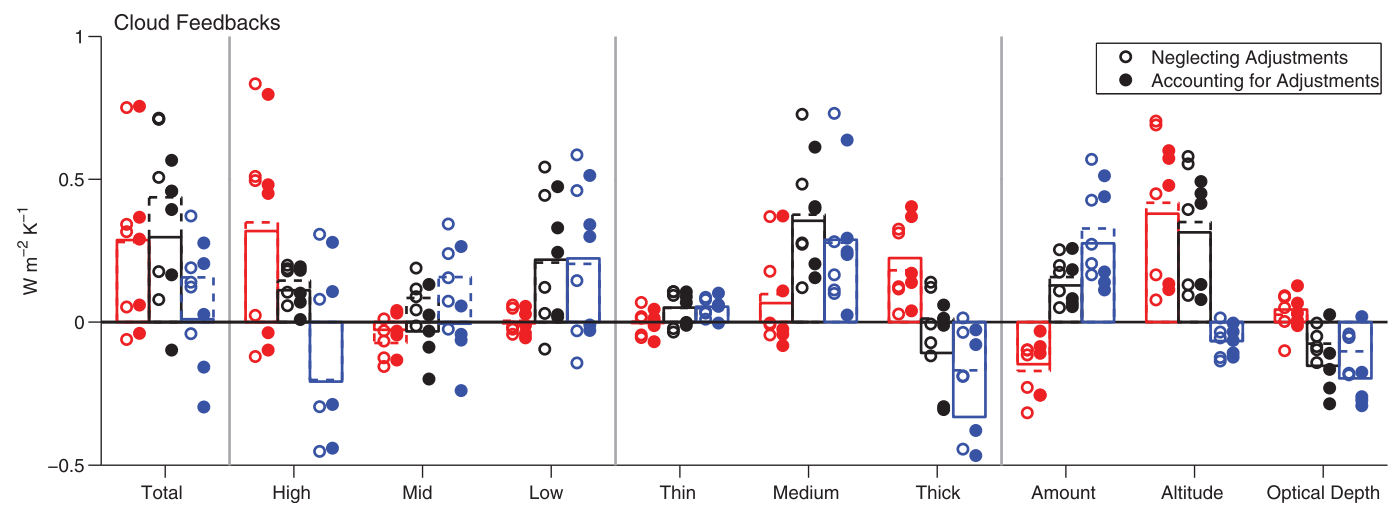

FIG. 3. As in Fig. 2, but for cloud feedbacks computed from the abrupt4xCO2 simulations. Kernel-derived feedbacks accounting for adjustments (indicated by filled circles and solid-line bars) are the slopes of the Gregory plots shown in Fig. 1. Kernel-derived feedbacks neglecting adjustments (indicated by unfilled circles and dashed-line bars) are the cloud-induced radiation anomalies averaged over the final $20 \mathrm{yr}$ of the simulation divided by the corresponding change in global mean surface temperature, and therefore rapid adjustments are aliased into these values, as described in section 5b. Numerical values of these feedbacks are provided in supplementary Tables 2 and 3. 

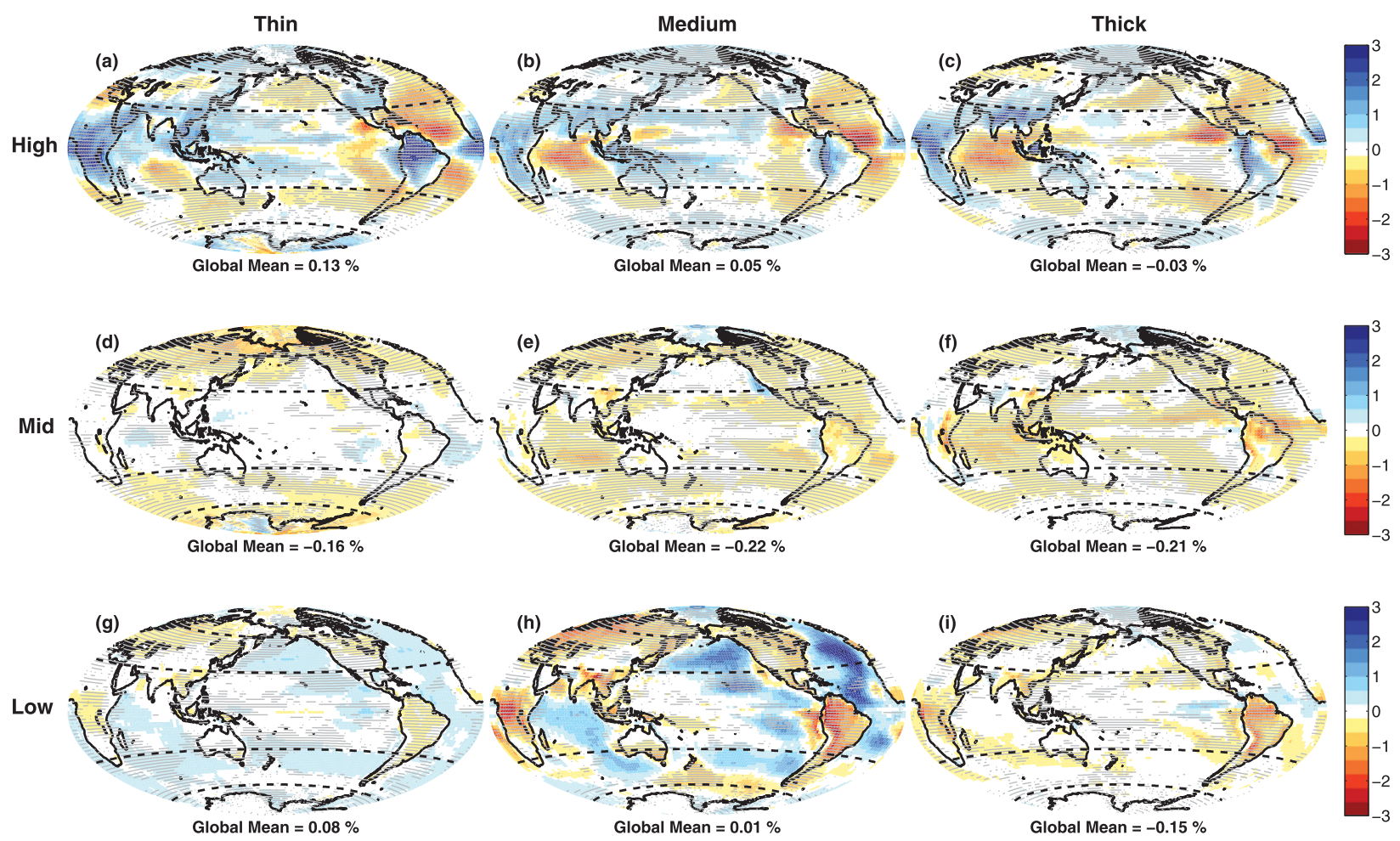

FIG. 4. Five-member ensemble mean rapid cloud adjustments diagnosed as the 30 -yr average cloud fraction anomaly (in absolute percent, not percentage change) from the fixed-SST experiment partitioned into nine standard ISCCP categories. Stippling indicates locations where at least four out of five models agree on the sign of the cloud anomalies, and the anomalies are not the result of obscuration effects (only relevant for midlevel and low-level clouds). Note that the five models averaged for this figure are not the exact same five models averaged for Fig. 5.

the results to the state of the climate at the time of quadrupling and provides a more robust and stable measure of the rapid cloud adjustment to $\mathrm{CO}_{2}$. It also does not rely on the assumption that the response evolves linearly with global mean temperature, as is the case when considering the regression line intercept. That the global mean anomalies in the fixed-SST experiment (red crosses in Fig. 1) tend to lie among the anomalies in the early stages of the abrupt $4 \mathrm{xCO} 2$ integration where they deviate most significantly from the regression line further supports their use as a more robust measure of the true cloud adjustment than the intercept. For these reasons, we have chosen to present the fixed-SST experiment anomalies, though we have performed the calculation with the other methods described above, and most features discussed below are similar, regardless of the chosen measure of rapid cloud adjustment.

Note that the rapid cloud adjustments described below are not occurring purely as a direct thermodynamic response to the $\mathrm{CO}_{2}$-induced change in atmospheric radiative heating rate in the presence of constant dynamics. Rather, clouds are responding to both rapid thermodynamic and dynamic changes, with the latter likely dominating at local scales. In the Hansen-style experiments we consider here, SSTs remain fixed but land surface temperatures are unconstrained; thus an anomalous land-sea temperature contrast develops, which creates an anomalous circulation to which the clouds are sensitive (Dong et al. 2009; Wyant et al. 2012; Cao et al. 2012). This is not uncharacteristic of transient climate change, as the ocean lags the land in heating up in the abrupt $4 \mathrm{xCO} 2$ experiments with a freely evolving ocean as well. Averaging over the entire tropics, Wyant et al. (2012), Watanabe et al. (2012), and Kamae and Watanabe (2013) find that the thermodynamic component of the $\mathrm{CO}_{2}$-induced cloud changes is dominant.

The five-member ensemble mean rapid cloud adjustments partitioned into high $(\mathrm{CTP} \leq 440 \mathrm{hPa})$, midlevel $(440 \mathrm{hPa}<\mathrm{CTP} \leq 680 \mathrm{hPa})$, low $(\mathrm{CTP}>680 \mathrm{hPa})$, thin ( $\tau \leq 3.6)$, medium-thickness $(3.6<\tau \leq 23)$, and thick $(\tau>23)$ cloud types following Rossow and Schiffer (1999) are shown in Fig. 4. For all figures hereafter, shades of blue will be used to indicate positive cloud amount, CTP, or $\tau$ anomalies, which tend to have a net 
cooling effect on the planet. Stippling is present only at locations where obscuration effects are deemed unimportant. The contributions of cloud fraction anomalies to the LW, SW, and net rapid cloud adjustments in these three altitude ranges and three optical depth ranges are shown in Fig. 2 and are provided in supplementary Table 1.

Although one might infer from Fig. 1 that the consistently small global mean LW rapid cloud adjustment is evidence that there is a negligible rapid high cloud response to $\mathrm{CO}_{2}$, it is clear from Fig. 4 that this is not true. High clouds of all optical thickness categories decrease significantly in the southern Indian Ocean, the eastern Pacific Ocean, and throughout the Atlantic Ocean, while increasing substantially over most land areas and over the Maritime Continent. In the global mean, thin and medium-thickness high clouds increase at the expense of high thick clouds, leading to a $0.15 \%$ increase in total high cloud fraction in the ensemble mean (sum across the top row of Fig. 4). The net high cloud radiative adjustment is systematically positive $\left(0.31 \pm 0.13 \mathrm{~W} \mathrm{~m}^{-2}\right)$ despite intermodel disagreement ${ }^{2}$ on whether LW or SW effects dominate (Fig. 2).

The largest global mean rapid adjustments occur for midlevel clouds, which exhibit a large global mean decrease of $0.59 \%$ (sum across the middle row of Fig. 4) and show systematic decreases in all thickness categories in almost every location (Fig. 4, middle).

Midlevel cloud reductions enhance the downwelling TOA net radiation by $0.53 \pm 0.27 \mathrm{~W} \mathrm{~m}^{-2}$, an amount that is equal to the combined high and low cloud contributions (Fig. 2; supplementary Table 1). Substantial midlevel cloud reductions are also apparent in previous studies [cf. Fig. 2a of Colman and McAvaney (2011) and Table 1 of Wyant et al. (2012)], but the rapid responses of low clouds have generally received more attention. Midlevel actual cloud fraction $\mathrm{cl}_{\text {actual }}$ (see appendix A) reductions are slightly greater in magnitude than those diagnosed by the simulator (not shown), suggesting that the reductions shown here are not overestimated because of simulator misassignment of clouds to midlevels. Though smaller than the overall decrease in midlevel clouds, the robust rapid decrease in highly reflective thick clouds of $0.39 \%$ (sum down the right column of Fig. 4), makes the single largest positive contribution to the net rapid cloud adjustment of all cloud types: $0.76 \mathrm{~W} \mathrm{~m}^{-2}$ in the ensemble mean (Fig. 2).

Over land, low clouds of all optical thickness categories decrease while high clouds increase. This dramatic

\footnotetext{
${ }^{2}$ Ensemble mean uncertainties represent the standard deviation across models.
}

upward shift in the cloud distribution is likely a response to the $\mathrm{CO}_{2}$-induced land-sea temperature contrast that forces anomalous ascent over land (e.g., Wyant et al. 2012; see also Fig. 6). The large reduction in low-level cloud over land is not an obscuration effect; in fact, the low $\mathrm{cl}_{\text {actual }}$ decreases over land are substantially larger than those diagnosed by the simulator. The large apparent increase in low clouds over ocean is, in most locations, entirely due to a substantial reduction in obscuration from midlevel and high clouds (note the lack of stippling). In contrast, low $\mathrm{cl}_{\text {actual }}$ anomalies are negative over vast portions of the ocean basins and are roughly $75 \%$ larger in magnitude than the reduction in midlevel $\mathrm{cl}_{\text {actual }}$. Nevertheless, the radiation reaching the top of the atmosphere depends primarily on the cloud tops that are actually visible from space; thus, despite the fact that low clouds decrease substantially upon quadrupling of $\mathrm{CO}_{2}$, large apparent increases in oceanic low cloud tops exposed to space will oppose the radiative impacts of coincident decreases in higher clouds. Indeed, in two models (HadGEM2-ES and MRI-CGCM3) the net low cloud radiative adjustment is negative (Fig. 2; supplementary Table 1). In the ensemble mean, however, decreases of thick low cloud that are roughly twice as large as increases in thin low cloud lead to a $0.06 \%$ decrease in the fraction of low clouds visible from space and a $0.22 \pm$ $0.44 \mathrm{~W} \mathrm{~m}^{-2}$ net radiative adjustment from low clouds (Fig. 2). As is the case for cloud feedback (Bony and Dufresne 2005; see below), the intermodel spread in net cloud radiative adjustment as a result of low clouds is greater than that resulting from clouds at any other altitude (Fig. 2).

\section{b. Spatial patterns of temperature-mediated cloud anomalies}

Maps of the local cloud response per unit change in global mean surface temperature are produced by regressing local cloud anomalies onto global mean surface temperature anomalies from the abrupt $4 \mathrm{xCO} 2$ runs. Multiplying these by the cloud radiative kernel gives the local contribution to the cloud feedback (section 4d). The five-member ensemble mean $\Delta T_{s}$-mediated cloud anomalies are shown in Fig. 5. The contributions of clouds in each of these three altitude ranges and three optical depth ranges to the global mean LW, SW, and net cloud feedbacks are shown in Fig. 3 and are provided in supplementary Table 2.

High clouds in all thickness categories show increases in the equatorial Pacific straddled to the north and south by negative anomalies, as convection shifts onto the equator. Negative high cloud anomalies are also evident over the tropical landmasses, a notable contrast from their rapid adjustment to $\mathrm{CO}_{2}$. High, thick cloud 

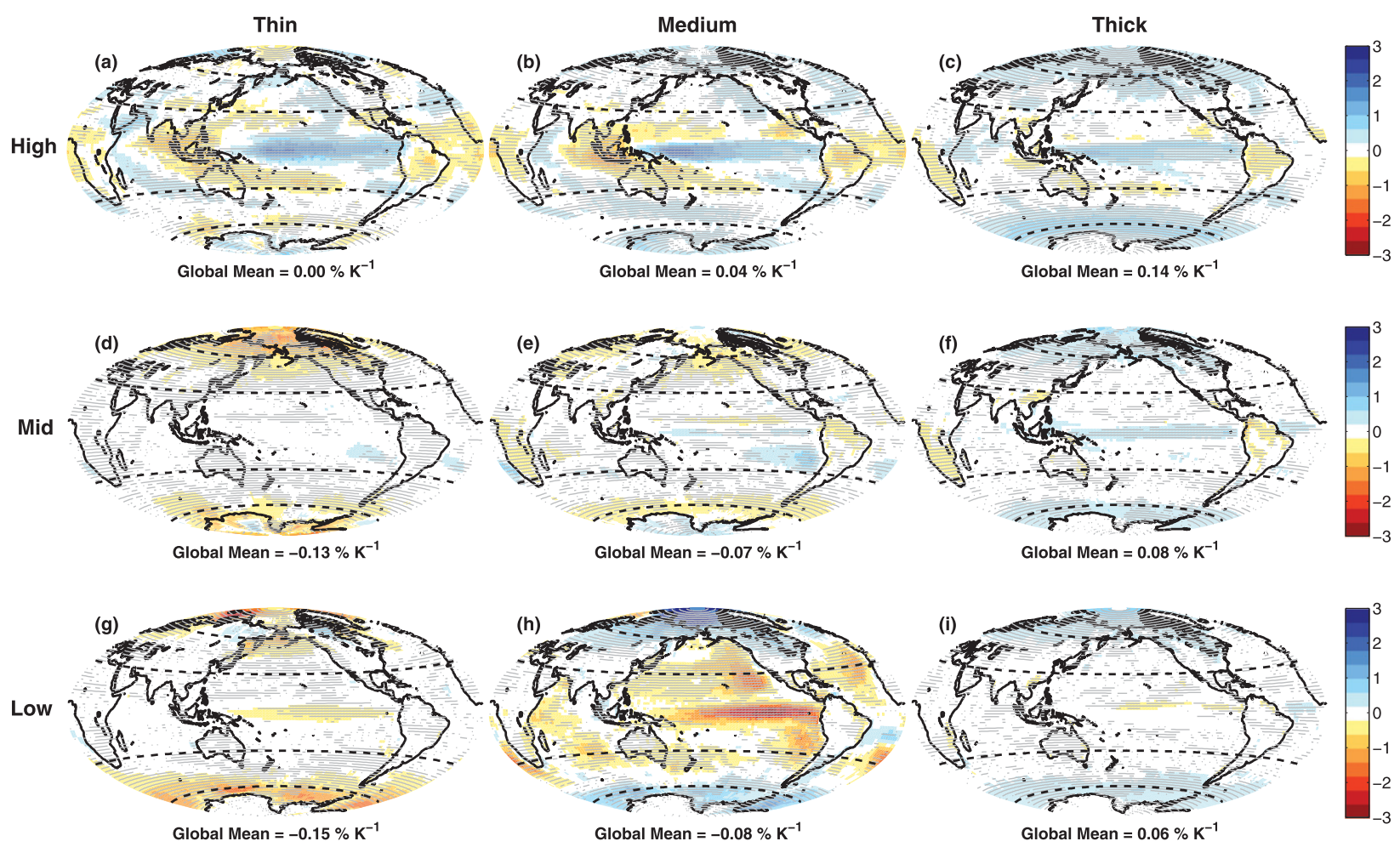

FIG. 5. Annual and ensemble mean $\Delta T_{s}$-mediated cloud anomalies partitioned into nine standard ISCCP categories. The values at each location represent the slope of the best-fit line of the local cloud fraction anomaly regressed on global mean surface temperature anomaly. Stippling indicates locations where at least four out of five models agree on the sign of the cloud anomalies, and the anomalies are not the result of obscuration effects. Note that the five models averaged for this figure are not the exact same five models averaged for Fig. 4.

fraction increases substantially in the global mean, especially over the Southern Ocean and in the high northern latitudes and deep tropics. These cloud changes contribute to a strong positive LW high cloud feedback of $0.32 \pm 0.38 \mathrm{~W} \mathrm{~m}^{-2} \mathrm{~K}^{-1}$ (Fig. 3; supplementary Table 2).

At midlevels, thin and medium-thickness clouds decrease while thick clouds increase in the global mean, though their anomalies and induced feedbacks are fairly small at every location. Thin and medium-thickness low clouds decrease in the global mean, with the former occurring primarily at high latitudes and the latter occurring throughout the ocean basins equatorward of about $60^{\circ}$.

Medium-thickness low clouds exhibit large reductions in coverage in every basin, especially over the stratocumulus regions and along the cold tongue in the Eastern Pacific. Although the decrease in stratocumulus clouds is robust, its magnitude varies considerably across models. The sign of these ISCCP simulator-produced low cloud anomalies is in good agreement with the actual model-produced low cloud anomalies, though the former generally has larger magnitudes in the stratocumulus regions and the eastern equatorial Pacific, suggesting some degree of overestimation of the reduction in low cloud amount owing to increases in obscuration by higher clouds. These changes are in striking contrast to the direct response to $\mathrm{CO}_{2}$ shown in Fig. 4 and lead to a robustly positive albeit widely varying low cloud feedback of $0.22 \pm 0.20 \mathrm{~W} \mathrm{~m}^{-2} \mathrm{~K}^{-1}$ (Fig. 3; supplementary Table 2).

In the ensemble mean, it is noteworthy that the increase in thick clouds and decrease in thin clouds are of the same magnitude $\left(0.28 \% \mathrm{~K}^{-1}\right)$ and that the increase in high clouds $\left(0.18 \% \mathrm{~K}^{-1}\right)$ is almost exactly equal to the decrease in low clouds $\left(0.17 \% \mathrm{~K}^{-1}\right)$, representing a marked shift of clouds from thin to thick types and from low to high types as the planet warms. Temperaturemediated changes to medium-thickness cloud types causes the single largest contribution to the positive net cloud feedback in the ensemble mean (Fig. 3), owing to the large decrease in medium-thickness low clouds over the low latitude oceans.

\section{c. Comparing rapid and temperature-mediated cloud anomaly patterns}

Figure 6 shows the zonal average rapid adjustments (left panels) and temperature-mediated anomalies (right 
(a) Land: Rapid $\Delta$ Cloud Amount (\%)

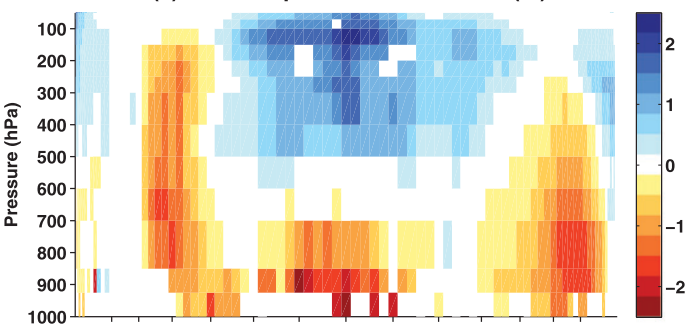

(b) Ocean: Rapid $\Delta$ Cloud Amount (\%)

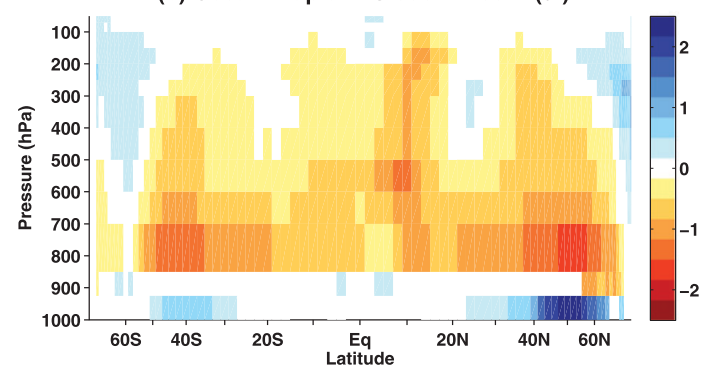

(c) Land: Ts-Mediated $\Delta$ Cloud Amount $\left(\% \mathrm{~K}^{-1}\right)$

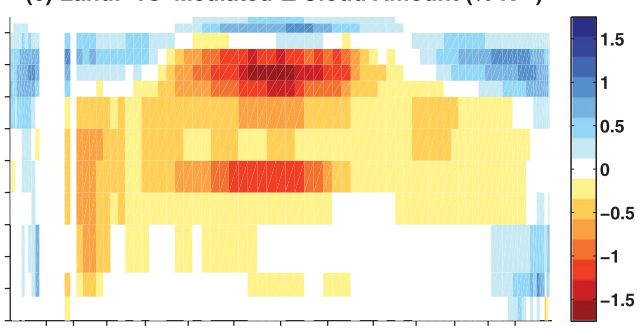

(d) Ocean: Ts-Mediated $\Delta$ Cloud Amount $\left(\% \mathrm{~K}^{-1}\right)$

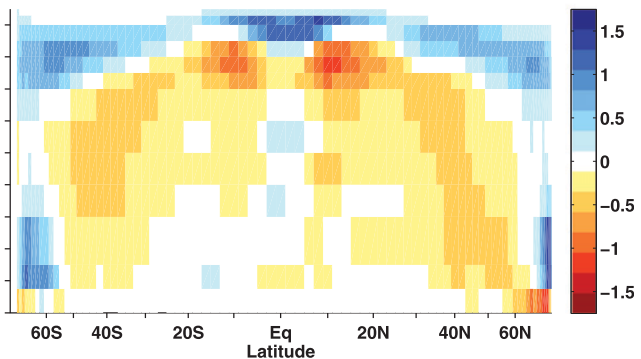

FIG. 6. Ensemble mean (left) rapid adjustments and (right) $\Delta T_{s}$-mediated anomalies in model-level cloud amount over (top) land and (bottom) ocean. Only locations in which at least four out of five models agree on the sign of the field are displayed. Model cloud fields are interpolated from their native grid to standard pressure levels prior to computing anomalies and averages. Note that the units and color bars in each column are different.

panels) in model-level cloud amount (i.e., the cloud amounts diagnosed by each model's cloud parameterization, not the ISCCP-simulator-produced clouds) separately for land (top panels) and ocean (bottom panels). We caution the reader to only compare the sign and spatial patterns of the rapid cloud responses to those of the $\Delta T_{s}$-mediated cloud responses, as the amount of cloud change due to $\mathrm{CO}_{2}$ relative to that due to $\Delta T_{s}$ depends on the magnitude of the $\mathrm{CO}_{2}$ perturbation and the realized amount of warming. In the fixed-SST experiment, land-based clouds equatorward of about $30^{\circ}$ latitude show large increases at pressures less than about $500 \mathrm{hPa}$, accompanied by decreases below this level (Fig. 6a). In contrast, oceanic clouds systematically decrease throughout the troposphere at pressures less than $850 \mathrm{hPa}$ and increase at the lowest levels (Fig. 6b). These features are likely as a result of the shift of convection from ocean to land following quadrupling of $\mathrm{CO}_{2}$, as the land heats up rapidly but the ocean does not, as shown in Wyant et al. (2012). Decreases in cloud amount throughout the entire free troposphere above $850 \mathrm{hPa}$ are especially pronounced between about $30^{\circ}$ and $60^{\circ}$ latitude in either hemisphere over both land and ocean. These large midlatitude cloud decreases may reflect a poleward expansion of the subtropical dry zones, which would be consistent with results attributing tropical widening to $\mathrm{CO}_{2}$ (Lu et al. 2009).

The sharp transition from positive to negative cloud fraction anomalies in the lower troposphere is more apparent when the individual models' cloud fields are plotted on their native vertical grids (Fig. 7). To emphasize stable regions over the low-latitude oceans dominated by stratiform clouds, we compute these averages over oceanic regions equatorward of $45^{\circ}$ latitude having values of lower-tropospheric stability [LTS; the difference between the potential temperature at 700 and $1000 \mathrm{hPa}$; Klein and Hartmann (1993)] in the top 20th percentile (exceeding about $15 \mathrm{~K}$ ). Above an LTS of $15 \mathrm{~K}$, low cloud amount dominates the total cloud amount (Wyant et al. 2009), though the results shown below are not sensitive to this threshold. It is clear from this figure that the level at which low cloud anomalies change sign closely tracks the level at which low cloud fractions peak, which varies from model to model. These features likely reflect the tendency for marine boundary layer clouds to descend as the boundary layer shoals (Watanabe et al. 2012; Wyant et al. 2012). Reductions in relative humidity associated with $\mathrm{CO}_{2}$-induced rapid warming at midlevels (Colman and McAvaney 2011; Kamae and Watanabe 2012) likely contribute to the negative cloud fraction anomaly above the top of the boundary layer.

Temperature-mediated cloud amount anomalies (right column of Fig. 6) are very different from the rapid adjustments described above. Unlike the fast adjustments, temperature-mediated cloud anomalies are quite similar for land and ocean. Cloud amounts decrease substantially throughout most of the troposphere equatorward of $60^{\circ}$ 

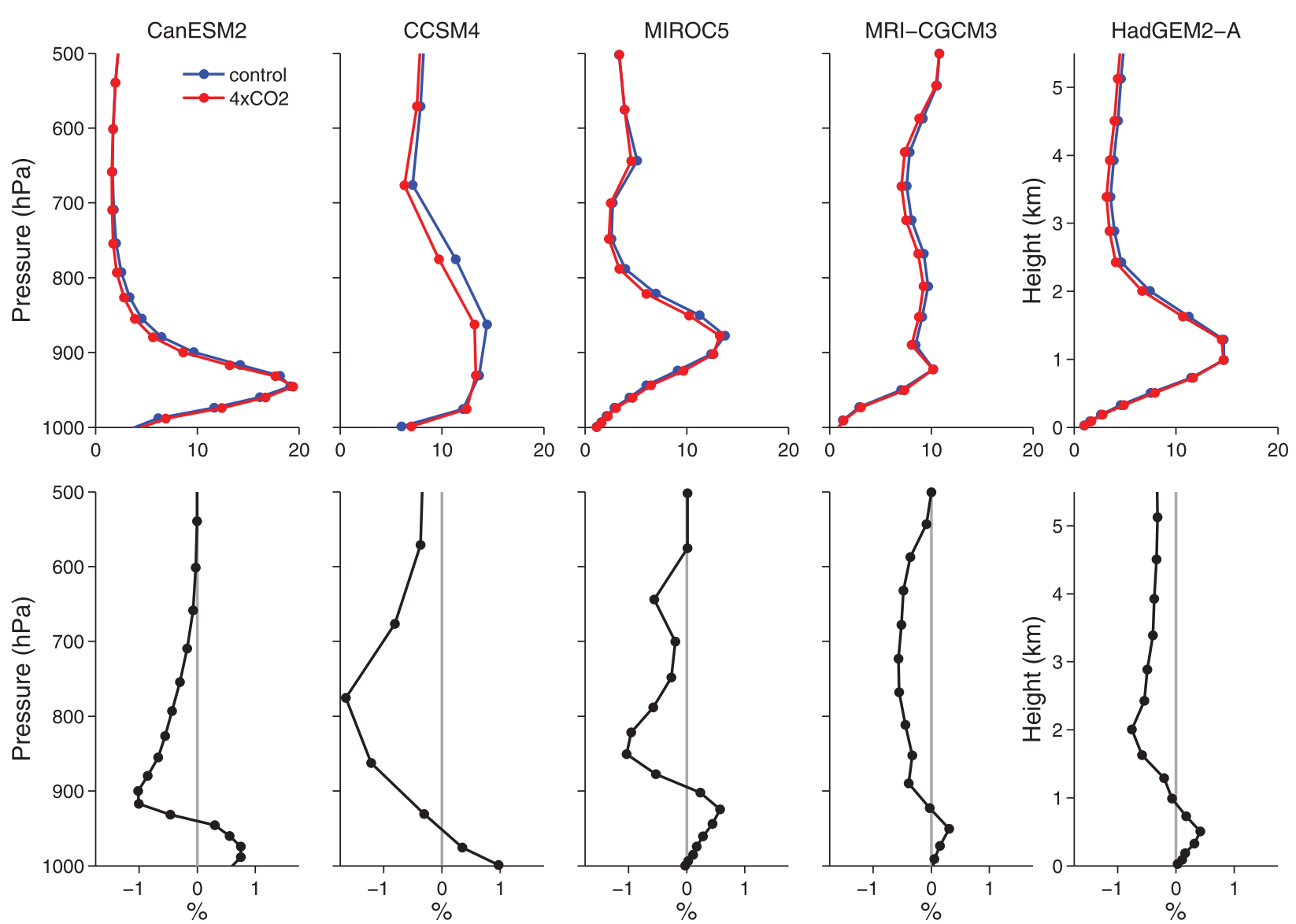

FIG. 7. (top) Mean cloud amounts for the sstClim (blue) and sstClim4xCO2 (red) simulations, averaged over regions equatorward of $45^{\circ}$ latitude having LTS values in the top 20th percentile. (bottom) The difference between the $4 \mathrm{xCO}_{2}$ and control-state cloud amounts shown in (top).

latitude, with no apparent compensating increases at low levels. An exception is the small increase in cloud amount at most altitudes on the equator over the ocean. A vertical dipole in upper-level cloud amount anomalies occurs at an altitude that transitions from about $150 \mathrm{hPa}$ in the tropics to about $400 \mathrm{hPa}$ at $60^{\circ}$ latitude. Poleward of $60^{\circ}$ latitude in both hemispheres, cloud amount anomalies are positive throughout the depth of the troposphere. These anomalies are suggestive of an upward shift of clouds at all latitudes as the troposphere deepens, and a poleward shift of midlatitude clouds as storm tracks and subtropical dry zones shift poleward. The pattern of cloud amount anomalies is essentially identical to that of relative humidity anomalies in models [cf. Fig. 2 of Sherwood et al. (2010)].

To synthesize the changes to cloud properties evident in Figs. 4-6, in Fig. 8 we show the changes in gross cloud properties for both the rapid responses to $\mathrm{CO}_{2}$ and the $\Delta T_{s}$-mediated responses that govern the cloud feedback. Upon $\mathrm{CO}_{2}$ quadrupling, cloud amount decreases everywhere except over Africa, Southeast Asia, Australia, the Arctic, and portions of the Pacific Ocean (Fig. 8a). Cloud top pressure decreases significantly over land upon $\mathrm{CO}_{2}$ quadrupling because of increased ascent over land but increases slightly over the subtropical oceans (Fig. 8b). The latter is because of an increase in the amount of lowlevel cloud tops exposed to space rather than a downward translation of cloud tops, although marine boundary layer cloud tops do descend (Fig. 7). A large reduction in $\tau$ occurs in response to quadrupled $\mathrm{CO}_{2}$ at most locations except over Africa, Southeast Asia, and the Arctic (Fig. 8c). This may be due to the significantly perturbed atmospheric energy budget following $\mathrm{CO}_{2}$ quadrupling: The reduction in atmospheric LW cooling would necessitate a reduction in latent heat release from condensation (Bala et al. 2010), which could plausibly lead to a reduction in cloud water and hence $\tau$, particularly in deep convective regions. Thus, clouds tend to become fewer, higher, and thinner upon quadrupling of $\mathrm{CO}_{2}$. The high spatial pattern correlation between rapid total cloud amount and altitude (optical 

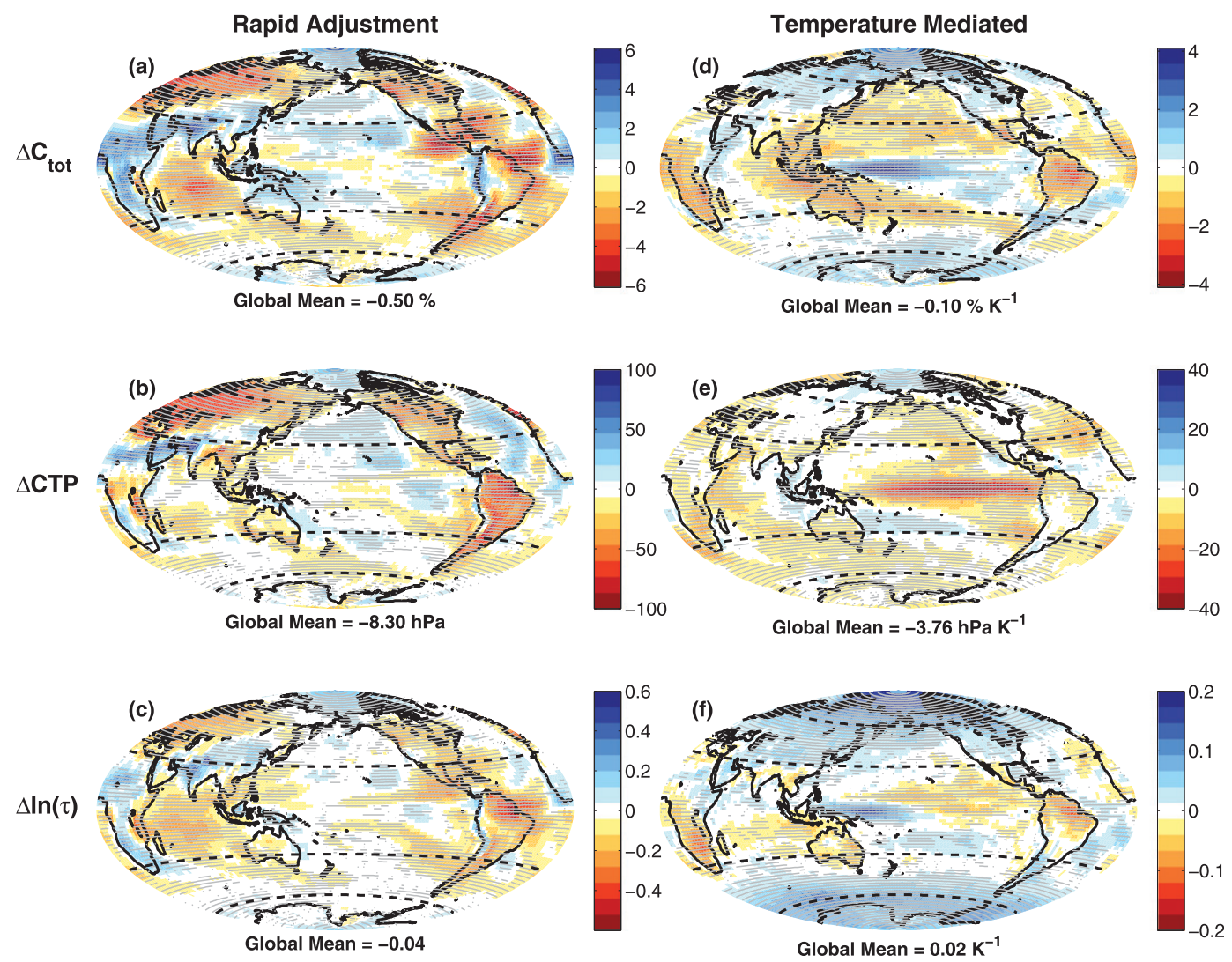

FIG. 8. Ensemble mean (left) rapid adjustments and (right) $\Delta T_{s}$-mediated cloud changes in (top) total cloud amount, (middle) CTP, and (bottom) logarithm of $\tau$. Note that the units and color bars in each panel are different. Stippling indicates locations where at least four out of five models agree on the sign of the field plotted.

depth) anomalies of $0.76(0.82)$ implies that these changes are very frequently coincident.

As the planet warms, the global mean cloud amount and CTP continue to decrease, but $\tau$ increases. Locally these changes are often of opposite sign to their rapid adjustment counterparts. Total cloud fraction decreases with increasing temperature in the subtropics and over tropical land areas and increases at higher latitudes and over the central and western Pacific (Fig. 8d). Cloud altitude increases everywhere except over the Arctic and some small regions of the tropical oceans where high clouds decrease (Fig. 8e). Cloud optical depth increases substantially in all extratropical regions, especially for cold clouds at high latitudes and altitudes (Fig. 8f). Decreases in cloud optical depth are confined to the tropics. All of these features are in close agreement with those shown in Fig. 1 of Zelinka et al. (2012b), suggesting that the temperature-mediated changes in gross cloud properties is qualitatively unchanged from those in CFMIP1. The pattern correlation of 0.79 between temperature-mediated changes in cloud amount and optical depth indicates that increases in cloud amount and optical depth frequently go hand in hand, as was the case for rapid adjustments. Unlike the rapid adjustments, patterns of temperature-mediated cloud altitude anomalies are poorly correlated with those of amount and optical depth, highlighting the relative uniformity of the altitude response in the face of large spatial variations in cloud amount and optical depth responses. Toggling between Figs. 4 and 5 and between the two columns in Figs. 6 and 8, it is clear that in some locations the cloud adjustments act in opposition to and in other locations act in the same direction as the cloud feedbacks. These features have important consequences for feedbacks that are computed without accounting for rapid cloud adjustments, as will be discussed in section $5 b$.

\section{d. Comparing patterns of cloud radiative adjustments and feedbacks}

Maps of the ensemble-mean TOA radiation anomalies resulting from rapid cloud adjustments and $\Delta T_{s}$-mediated 

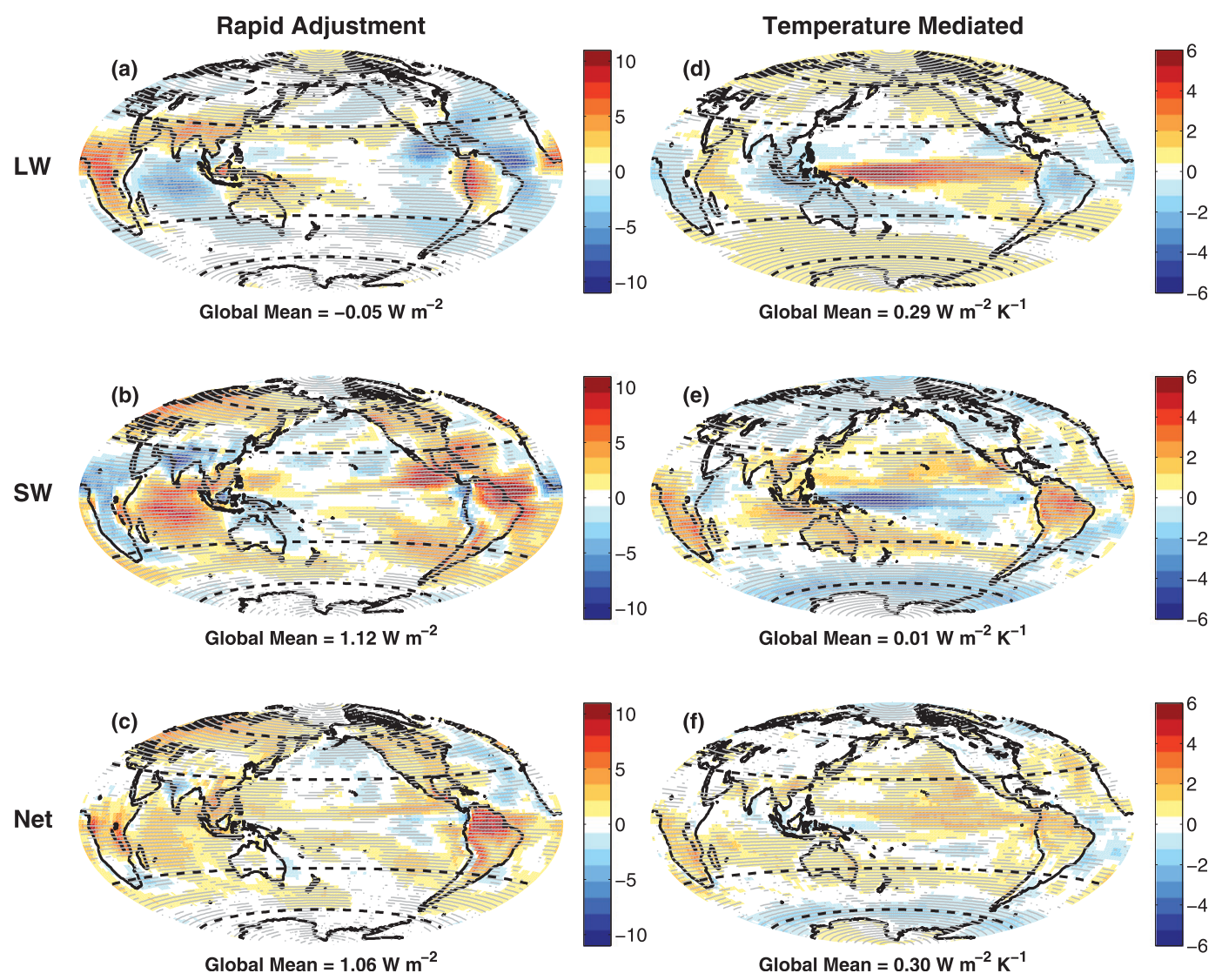

FIG. 9. Ensemble mean (top) LW, (middle) SW, and (bottom) net radiation anomalies as a result of (left) rapid cloud adjustments and (right) $\Delta T_{s}$-mediated cloud changes. Note that the units and color bars in each column are different. Stippling indicates locations where at least four out of five models agree on the sign of the field plotted.

cloud anomalies are shown in Fig. 9. Following the decomposition of cloud anomalies introduced in Zelinka et al. (2012b) but with modifications explained in appendix $\mathrm{B}$, we compute the contributions of changes in cloud amount, altitude, and optical depth to the LW, SW, and net cloud feedbacks and rapid adjustments. ${ }^{3}$ The global mean LW and SW radiation anomalies as a result of these gross cloud property changes are shown in Figs. 2 and 3 and are provided in supplementary Tables 1 and 2.

LW heating from rapid increases in cloud-top altitude over land is exceeded by LW cooling from widespread rapid decreases in cloud amount and optical depth, making the ensemble and global mean LW rapid cloud

\footnotetext{
${ }^{3}$ One must bear in mind that such a decomposition can sometimes be misleading (e.g., large reductions solely in low clouds can cause a large positive LW cloud altitude feedback when such low cloud anomalies would have little actual effect on LW fluxes at the TOA).
}

adjustment negative but very small in magnitude (Fig. 9a). In contrast, ensemble mean cloud-induced LW anomalies increase linearly with increasing global mean surface temperature because the LW heating effect of higher and thicker clouds exceeds the LW cooling effect of fewer clouds. Only over portions of the tropics that have particularly large high cloud amount reductions is the local LW cloud feedback negative (Fig. 9d). The LW cloud altitude feedback is robustly positive and is supplemented in four out of five models by a smaller positive LW cloud optical depth feedback (Fig. 3; supplementary Table 2). Thus, in this ensemble of five models, nearly all of the enhanced LW heating because of clouds is attributable to LW cloud feedback rather than to abrupt cloud changes, in agreement with Colman and McAvaney (2011) and Andrews et al. (2012a). It is important to recall, however, that even in the ensemble mean there are large local instantaneous LW cloud anomalies.

Changes in cloud-top altitude have little influence on reflected SW radiation, but large rapid reductions in 
cloud amount and optical depth cause large reductions in reflected SW radiation over much of the globe, contributing an additional $1.12 \mathrm{~W} \mathrm{~m}^{-2}$ to the $\mathrm{CO}_{2}$ forcing in the ensemble and global mean (Fig. 9b). In contrast, temperature-mediated decreases in total cloud amount and cloud optical depth at low latitudes (except over the equatorial Pacific) are opposed by large increases in cloud optical depth (and in some regions, total cloud amount) at higher latitudes. The net result is a near-zero global and ensemble mean SW cloud feedback (Fig. 9e). Thus, in the ensemble mean, all of the enhanced absorbed SW radiation arises purely from abrupt cloud changes rather than as a steadily increasing SW absorption anomaly over the course of the run. This is opposite to the LW case and is quite similar to the results of Gregory and Webb (2008), Andrews and Forster (2008), and Andrews et al. (2012a). Recall, however, that the global mean SW cloud feedback can be large within individual models (Fig. 3) and that large regional contributions exist even in models with zero global mean SW cloud feedback.

The ensemble mean net cloud radiative adjustment is quite strongly positive because of nearly equal contributions from decreases in amount, CTP, and $\tau$ (Fig. 2), while the positive net cloud feedback arises from positive amount and altitude feedbacks, opposed by a negative optical depth feedback. The increase in cloud optical depth is the only negative contributor to the net cloud feedback in the ensemble mean (Fig. 3). It is negative in all but the MRI-CGCM3 model, in which it is statistically indistinguishable from zero (supplementary Table 2). It is noteworthy that both the net cloud radiative adjustment and feedback maps (Figs. 9c,f, respectively) are dominated by positive values in most regions.

We compare the results shown here to those from the 11 CFMIP1 slab ocean models analyzed by Zelinka et al. (2012a,b) but note that models from different centers and a different number of models are included in the two ensembles, that CFMIP1 models used slab oceans whereas CFMIP2 models used fully dynamic oceans, and that rapid adjustments could not be accounted for in CFMIP1. To address this final discrepancy, we can compute the CFMIP2 feedbacks neglecting adjustments (category III in Table 1; see section 5b). These are shown as unfilled circles in Fig. 3. In the following, we discuss only those aspects that are insensitive to whether kernelderived CFMIP2 feedbacks neglecting adjustments or accounting for adjustments are compared to the CFMIP1 feedbacks.

Feedbacks from thick, midlevel, and low cloud types are notably smaller in CFMIP2 than in CFMIP1 (not shown). A positive ensemble mean net adjustment arises from changes in these three cloud types in CFMIP2, suggesting that some portion of the positive thick, midlevel, and low cloud feedbacks diagnosed in CFMIP1 were actually because of rapid cloud reductions. The feedback from thin, medium-thickness, and high cloud types and the altitude and optical depth feedbacks are essentially unchanged between the two ensembles. Temperature-mediated reductions in total cloud amount are smaller in CFMIP2 than in CFMIP1 (not shown); thus, the amount feedback is less positive in CFMIP2. Moreover, clouds are optically thinner in the mean state in CFMIP2 than in CFMIP1 (Klein et al. 2013). In a model with thinner clouds, a given cloud amount decrease will cause a smaller reduction in planetary albedo (i.e., a less positive cloud amount feedback), all else being equal.

As in CFMIP1, the intermodel spread in LW and SW high cloud feedback is much larger than that resulting from low clouds (standard deviations of 0.4 versus $0.2 \mathrm{~W} \mathrm{~m}^{-2} \mathrm{~K}^{-1}$ ), but compensation between the LW and SW effects causes the intermodel spread in net cloud feedback to be dominated by low clouds (Fig. 3; supplementary Table 2). As in CFMIP1, medium-thickness cloud reductions are the single largest contributor to the positive net cloud feedback and contribute positively in every model.

\section{Implications of diagnostic and methodological choices}

\section{a. Sensitivity to diagnostics}

Comparing the gray and black points in Fig. 1, it is clear that LWCRE anomalies are systematically more negative or less positive than cloud-induced LW flux anomalies, and this difference increases as the climate warms in every model. In Fig. 10, we show the cloudinduced LW anomaly, the LWCRE anomaly, and their difference, averaged across the five fixed-SST experiments. The global mean instantaneous LWCRE anomalies (Fig. 10b) are quite strongly negative, from which one might infer a large cloud response to $\mathrm{CO}_{2}$ that reduces the forcing due to $\mathrm{CO}_{2}$ (Gregory and Webb 2008). In contrast, the global mean kernel-derived LW cloud adjustment is quite small, though it locally exhibits large values of either sign (Fig. 10a). The difference map between cloud-induced LW flux anomalies and LWCRE anomalies (Fig. 10c) exhibits relatively uniform positive values that closely tracks the mean-state high cloud distribution [see also Fig. 1 of Wyant et al. (2012)]. This difference map provides an estimate of the so-called cloud masking of the radiative perturbations arising primarily from quadrupled $\mathrm{CO}_{2}$ concentrations. The masking arises because increases in $\mathrm{CO}_{2}$ cause a larger decrease in upwelling LW fluxes in cloud-free than 
(a) LW Cloud Adjustment (Kernel)

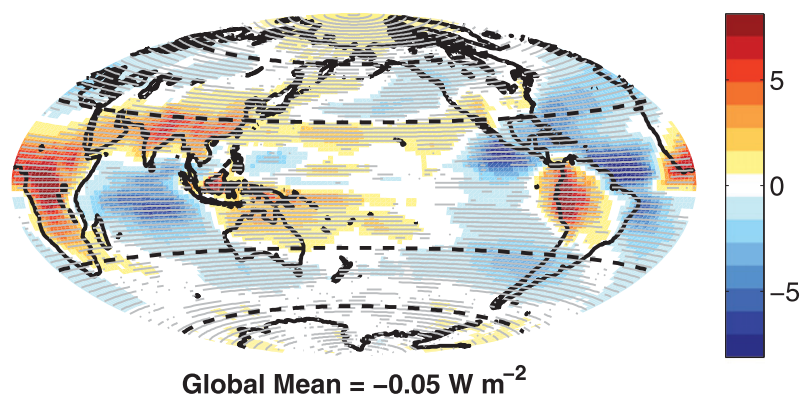

(b) LW Cloud Adjustment (CRE)

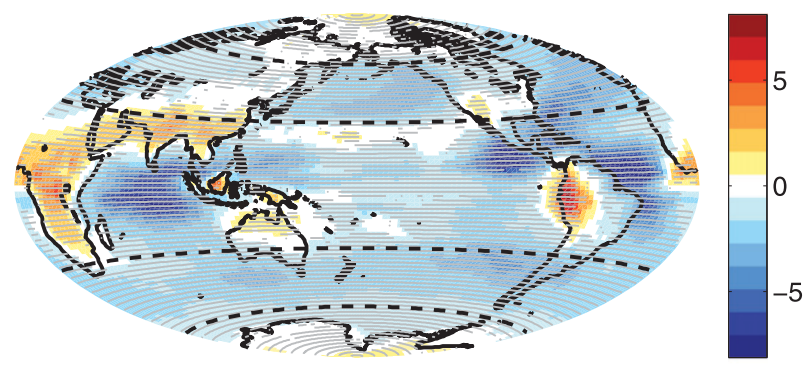

Global Mean $=-1.37 \mathrm{~W} \mathrm{~m}^{-2}$

(c) Difference $=$ Masking

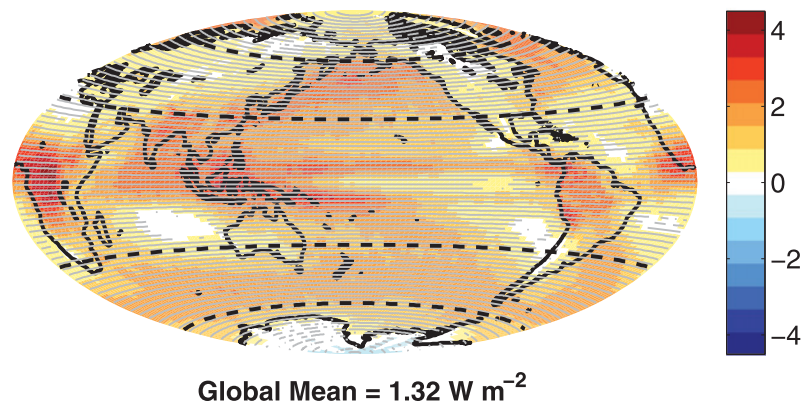

FIG. 10. Ensemble mean (a) kernel-derived and (b) CREderived LW rapid cloud adjustments diagnosed from the fixed-SST experiments, along with (c) their difference. Note that the color bars in (a) and (b) range from -8 to $8 \mathrm{~W} \mathrm{~m}^{-2}$, whereas that in (c) ranges from -4.5 to $4.5 \mathrm{~W} \mathrm{~m}^{-2}$. Stippling indicates locations where at least four out of five models agree on the sign of the field plotted.

in overcast conditions, thereby reducing LWCRE independently of any cloud-induced radiation changes. The LW anomalies that are actually because of rapid cloud changes are captured in the kernel-derived estimates (Fig. 10a). We derive an ensemble mean LW masking of roughly $1.3 \mathrm{~W} \mathrm{~m}^{-2}$, quite close to estimates given in Soden et al. (2008), Colman and McAvaney (2011), and Andrews et al. (2012a).

In contrast to the LW cloud adjustments, $\triangle$ SWCREderived and SW kernel-derived rapid cloud adjustments are much closer to each other (note the overlapping red crosses in the right column of Fig. 1) and their small difference (cloud masking of the SW forcing) varies in sign across models. Whether the presence of clouds increases or decreases the small SW radiative forcing from $4 \mathrm{xCO}_{2}$ depends on the impact of clouds on the pathlength of solar photons relative to cloud-free conditions, which varies among the models depending on various factors including cloud height and optical depth, how multiple scattering is treated, and the underlying surface albedo.

The use of CRE as a diagnostic also has implications for computing cloud feedback, for essentially the same reasons. Because CRE anomalies can be caused by changes in noncloud fields, the change in CRE with temperature is in general not the same as the cloud feedback, as discussed in Soden et al. $(2004,2008)$. The difference between cloud feedbacks computed with $\triangle \mathrm{CRE}$ and with cloud radiative kernels (categories II and IV, respectively, in Table 1) is apparent in Fig. 1 (cf. slopes of black and gray symbols). Averaged across all five models, the kernel-derived net cloud feedback is roughly $0.3 \mathrm{~W} \mathrm{~m}^{-2} \mathrm{~K}^{-1}$ greater than that derived with net $\triangle \mathrm{CRE}$ (not shown) and is positive rather than negative. Only one out of five models in this study (MIROC5) has a negative net cloud feedback, whereas three have negative feedbacks when computed with CRE. The difference between these two measures of cloud feedback gives an estimate of the cloud masking of the noncloud feedbacks. Large positive values over the high and low latitudes originate from clouds masking the surface albedo and water vapor feedbacks, respectively (not shown). Soden et al. (2008) derive a global mean masking value of $0.66 \mathrm{~W} \mathrm{~m}^{-2} \mathrm{~K}^{-1}$ in CMIP3 models running the Special Report of Emissions Scenarios (SRES) A1b scenario, roughly twice as large as that derived here, but with a very similar spatial pattern. Smaller masking of the noncloud feedbacks in the CMIP5/CFMIP2 ensemble may be as a result of mean-state clouds being optically thinner than in CMIP3/CFMIP1 (Klein et al. 2013).

\section{b. Sensitivity to methodology}

Before it was recognized that clouds may undergo a rapid adjustment in direct response to increased $\mathrm{CO}_{2}$, all cloud changes that existed at the end of a perturbed $\mathrm{CO}_{2}$ simulation were assumed to have occurred in response to increasing surface temperature and therefore were incorporated into the feedback. One would simply take the average cloud-induced radiation anomalies at the end of the run and divide by the corresponding change in global mean surface temperature to compute the feedback. This is equivalent to computing the feedback as the slope of the regression line passing through $(0,0)$ and the mean value of the points at the end of the run in Fig. 1. 
In this section, we compare kernel-derived cloud feedbacks neglecting adjustments and those computed accounting for adjustments (categories III and IV, respectively, in Table 1). Note that the difference between these two estimates will decrease as the length of the run increases toward a new equilibrium state. Moreover, the cloud feedback computed neglecting adjustments will differ among models that have identical cloud adjustments and feedbacks if they have different magnitudes of $\Delta T_{s}$. Kernel-derived cloud feedbacks neglecting adjustments computed using anomalies from the final $20 \mathrm{yr}$ of the abrupt $4 \mathrm{xCO} 2$ run are indicated with unfilled circles and dashed bars in Fig. 3 and are given in supplementary Table 3 .

The net cloud feedback computed neglecting adjustments is about $50 \%$ larger than that computed accounting for adjustments in this ensemble. Most of the difference between the two feedback estimates arises from the SW component. Whereas the ensemble mean SW cloud feedback is $0.01 \mathrm{~W} \mathrm{~m}^{-2} \mathrm{~K}^{-1}$ when accounting for adjustments, it is $0.16 \mathrm{~W} \mathrm{~m}^{-2} \mathrm{~K}^{-1}$ when they are neglected (Fig. 3; supplementary Tables 2 and 3). When accounting for adjustments, the midlevel and thick SW cloud feedbacks are 0.17 and $0.16 \mathrm{~W} \mathrm{~m}^{-2} \mathrm{~K}^{-1}$ lower, respectively, than their adjustment-neglected counterparts because of the rapid $\mathrm{CO}_{2}$-induced reduction in midlevel and thick clouds (Fig. 4). Also notable is that the SW optical depth feedback is much less positive at low latitudes and much more negative at high latitudes when adjustments are accounted for (not shown). As found in Zelinka et al. (2012b), the large negative net cloud feedback over the Southern Ocean comes from the shift toward thicker clouds and increase in total cloud amount, but the former is roughly 4 times stronger in the zonal mean at $60^{\circ} \mathrm{S}$. Properly accounting for the rapid cloud adjustments further increases the importance of cloud brightening over cloud increases in causing the high-latitude negative feedback.

\section{Conclusions}

In this paper, we have analyzed and described the direct responses of clouds to an abrupt quadrupling of $\mathrm{CO}_{2}$ in five CMIP5/CFMIP2 GCMs as well as the subsequent changes in clouds that progress as the planet warms. In addition, we have used cloud radiative kernels to quantify the radiative impact of these cloud anomalies, thereby providing the first simultaneous model intercomparison of cloud feedbacks and rapid cloud radiative adjustments, partitioned among changes in various cloud types and in the overall amount, altitude, and optical depth of clouds, with no influence from cloud masking effects.
A spatially uniform decrease in midlevel clouds, shift from thicker to thinner cloud types, increase (decrease) in high (low) clouds over land, and decrease and descent of low-latitude marine stratiform clouds initially occurs upon quadrupling of $\mathrm{CO}_{2}$. Though in every model these cloud anomalies have only a small negative (cooling) influence on the global mean LW budget of the planet, they strongly increase the amount of SW radiation absorbed by the planet, consistent with many previous studies (Andrews and Forster 2008; Colman and McAvaney 2011; Watanabe et al. 2012; Andrews et al. 2012a; Kamae and Watanabe 2012). The intermodel spread in net cloud radiative adjustment as a result of low clouds is greater than that resulting from clouds at any other altitude. Abrupt reductions in cloud amount, cloud-top pressure, and optical depth make roughly equal contributions to the $1.06 \mathrm{~W} \mathrm{~m}^{-2}$ net enhancement of $4 \mathrm{xCO}_{2}$ forcing. Some of these responses (e.g., the increase in high clouds over land) are likely driven by the significant land-ocean difference in warming and the attendant circulation changes, as warming over land is unconstrained in these experiments. Thus, one should not think of the rapid cloud anomalies as being solely as a result of $\mathrm{CO}_{2-}$ induced changes in the clouds' thermodynamic environment but rather a combination of dynamic and thermodynamic changes.

As the planet warms because of quadrupled $\mathrm{CO}_{2}$ levels, cloud-top altitude increases at nearly every location, leading to a large positive LW cloud feedback, consistent with Zelinka and Hartmann (2010) and Zelinka et al. (2012b). Low clouds equatorward of $60^{\circ}$ decrease substantially over every ocean basin as the planet warms, and cold clouds at high latitudes and altitudes become thicker, leading to positive SW cloud amount and negative SW cloud optical depth feedbacks, as also found in CFMIP1 models (Zelinka et al. 2012b). In the ensemble mean, all of the global mean cloudenhanced SW heating that is present at the end of the abrupt $4 \mathrm{xCO} 2$ simulation arose from cloud reductions immediately upon introduction of the forcing agent, with little $\Delta T_{s}$-mediated response, though this may not be true in individual models. In contrast, nearly all of the global mean cloud-enhanced LW heating present at the end of the abrupt $4 \mathrm{xCO} 2$ simulation in every model arose from $\Delta T_{s}$-mediated cloud-top altitude increases, with little direct response to the forcing agent.

We have also highlighted the implications of diagnostic and methodological choices on the derived cloud feedbacks and radiative adjustments. First, we showed that CRE-derived LW cloud adjustments are strongly negatively biased, owing to the $1.3 \mathrm{~W} \mathrm{~m}^{-2}$ masking of the $4 \mathrm{xCO}_{2}$ radiative forcing by clouds rather than the actual cloud adjustment to $\mathrm{CO}_{2}$. Similarly, the CRE-derived 
net cloud feedback is negatively biased because of roughly $0.3 \mathrm{~W} \mathrm{~m}^{-2} \mathrm{~K}^{-1}$ cloud masking of positive noncloud feedbacks. Second, we showed that calculating cloud feedbacks by simply taking the cloud-related radiation anomalies at the end of a perturbed run and dividing by the corresponding global mean surface temperature anomaly, as is commonly done, results in a $50 \%$ overestimate of the global mean net cloud feedback in this ensemble of five models. This is because of the large adjustments that occur immediately upon $\mathrm{CO}_{2}$ quadrupling that are better interpreted as an adjustment to the forcing than as a feedback. These primarily affect the SW cloud feedbacks, for which failure to account for rapid adjustments leads to an overestimate of the positive amount feedback and an underestimate of the magnitude of the negative optical depth feedback.

Our primary purpose in this paper was to detail the cloud anomalies responsible for rapid adjustments and feedbacks across an ensemble of currently available CMIP5/CFMIP2 models and to quantify the effect of different methodological and diagnostic choices on the derived cloud feedbacks and rapid radiative adjustments. We have not attempted to explain every feature that is present in the results and hope that this paper will motivate further study of these cloud processes. Specifically, this study has raised numerous questions, like the following:

- Does the reduction in atmospheric radiative cooling immediately following $\mathrm{CO}_{2}$ quadrupling cause the abrupt decrease in cloud optical depth?

- Are the large midlatitude cloud reductions between 200 and $800 \mathrm{hPa}$ in each hemisphere in the fixed-SST experiments evidence of a poleward shift of the storm tracks because of $\mathrm{CO}_{2}$ alone?

- Are the rapid midlevel cloud reductions solely due to a decrease in relative humidity of the middle troposphere because of the reduced radiative cooling from increased $\mathrm{CO}_{2}$ ?

- What causes the large deviations from linearity evident in the early stages of the quadrupled simulations in some models but not in others?

As a follow up to the final question, we note that, although global mean cloud-induced radiation anomalies behave remarkably linearly for the majority of abrupt forcing simulations, the linear forcing-feedback paradigm cannot fully capture the rich structure evident in the time-evolving radiative anomalies. Moreover, separation between rapid $\left(\mathrm{CO}_{2}\right.$ adjustment) and slow $\left(\Delta T_{s}\right.$ mediated) time scales is not clear and likely varies from model to model. Armour et al. (2013) make a compelling case that the apparent time dependence of feedbacks can be explained by the actuation of time-invariant locally defined feedbacks by surface warming patterns that evolve on several time scales. Clearly, consideration of the time-evolving radiative anomalies is crucial for properly understanding the role of clouds in altering the radiation budget of the perturbed climate.

Acknowledgments. We thank three anonymous reviewers for their thoughtful criticisms of this paper. We acknowledge the World Climate Research Programme's Working Group on Coupled Modelling, which is responsible for CMIP, and we thank the climate modeling groups (listed in Table 2) for producing and making available their model output. For CMIP, the U.S. Department of Energy's (DOE) Program for Climate Model Diagnosis and Intercomparison provides coordinating support and led development of software infrastructure in partnership with the Global Organization for Earth System Science Portals. The work of MDZ, SAK, and KET was supported by the Regional and Global Climate Modeling Program of the Office of Science at the DOE and was performed under the auspices of the DOE by Lawrence Livermore National Laboratory under Contract DE-AC52-07NA27344. TA, MJW, and JMG were supported by the Joint DECC/Defra Met Office Hadley Centre Climate Programme (GA01101). MJW is also supported by funding from the European Union Seventh Framework Programme (FP7/2007-2013) under Grant Agreement 244067 via the EU Cloud Intercomparison and Process Study Evaluation project (EUCLIPSE).

\section{APPENDIX A}

\section{Determining Obscuration-Affected Cloud Fraction Anomalies}

To verify that the sign of the high, midlevel, and low cloud fraction changes seen by the simulator are "real" and are not coming from obscuration effects, we have computed "actual" cloud fraction anomalies $\Delta \mathrm{cl}_{\text {actual }}$ in each altitude range. Actual cloud fractions $\mathrm{cl}_{\mathrm{actual}}$ are computed by summing (with random overlap) the model-produced cloud amount $\mathrm{cl}$, ignoring any values outside of the altitude range of interest. As $\Delta \mathrm{cl}_{\text {actual }}$ is unaffected by any overlying cloud fraction anomalies, sign disagreements between it and the simulatorobserved cloud anomalies are likely due to obscuration effects. Obscuration-affected cloud fraction anomalies $\Delta \mathrm{cl}_{\text {space-view }}$ are computed by vertically summing cl but accounting for random overlap with values above the altitude range of interest. Thus, if cl equals $100 \%$ at $850 \mathrm{hPa}$ but is overlain by a cl of $75 \%$ at $250 \mathrm{hPa}$, low $\mathrm{cl}_{\text {actual }}$ equals $100 \%$ whereas low $\mathrm{cl}_{\text {space-view }}$ equals $25 \%$. Locations in which simulator-observed cloud anomalies 
are purely a result of changes in obscuration are identified as any location where the sign of the simulatorobserved cloud anomaly disagrees with that of $\Delta \mathrm{cl}_{\text {actual }}$ but agrees with the sign of $\Delta \mathrm{cl}_{\text {space-view }}$

The comparison is not perfect, since $\mathrm{cl}$ is nonzero throughout the depth of a cloud whereas the simulator diagnoses cloud tops, and errors are introduced when assuming random overlap statistics with monthly resolved data. Such uncertainties preclude us from quantitatively comparing magnitudes of the three types of cloud anomalies. It is likely that - even where the signs of the simulatorobserved cloud anomaly and $\Delta \mathrm{cl}_{\text {actual }}$ agree-obscuration effects are having an impact. We expect these obscuration effects to apply indiscriminately to all optical depths, such that the optical depth-dependence of cloud anomalies discussed in section 4 is likely real, even in the presence of obscuration effects.

\section{APPENDIX B}

\section{A Modified Decomposition of Cloud-Induced Radiation Anomalies}

Zelinka et al. (2012b) proposed a decomposition of the anomalous cloud fraction histogram that allowed for partitioning the cloud-induced radiative flux anomalies $\Delta R_{C}$ into contributions that account for the change in total cloud amount, the change in the vertical distribution of clouds, and the change in the cloud optical depth distribution. The sum of these three components would ideally sum to the total $\Delta R_{C}$, but in general a residual remains because the full variations found in the cloud histogram are too complex to be expressed as a simple sum of three terms.

As an alternative, we describe here how the radiative kernel, rather than changes in cloud fraction, can be resolved into components that better isolate these three contributions to total $\Delta R_{C}$. One might expect that, since compared with cloud fraction changes the radiative kernel more consistently varies with optical depth (across all cloud-top temperatures) and with cloud-top temperature (across all optical depths), a smaller residual might result from this approach. This will indeed turn out to be the case.

We shall proceed by first resolving the cloud fraction anomaly into two terms and then resolving the radiative kernel into four terms. This will result in a four-term decomposition of $\Delta R_{C}$.

We express the cloud fraction anomaly as

$$
\Delta C_{p \tau}=\left(\frac{C_{p \tau}}{C_{\text {tot }}}\right) \Delta C_{\text {tot }}+\Delta C_{p \tau}^{*},
$$

where total cloud cover, accounting for contributions from all $\mathrm{CTP}-\tau$ categories, is given by

$$
C_{\text {tot }}=\sum_{p=1}^{P} \sum_{\tau=1}^{T} C_{p \tau}
$$

The first term on the rhs of (B1) represents the contribution to $\Delta C_{p \tau}$ from a hypothetical change in total cloud cover that is apportioned across the CTP $-\tau$ categories in such a way as to leave the original normalized distribution unaltered. Thus, the first term accounts for the effects of a change in cloud cover, holding fixed the distribution across CTP and $\tau$ categories, and is identical to "the proportionate change in cloud fraction" derived in Zelinka et al. (2012b). The second term on the rhs of (B1) accounts for shifts in the distribution of altitudes and optical depths of clouds, with total cloud fraction held fixed. By construction, this term will vanish when the equation is summed over all CTP- $\tau$ categories.

Next, we resolve the radiative kernel as the sum of two terms,

$$
K_{p \tau}=K_{0}+K_{p \tau}^{\prime}
$$

Here, $K_{0}$ is an average over the individual CTP $-\tau$ categories of the radiative kernel, weighted by the fraction of total cloud cover accounted for by each category,

$$
K_{0}=\sum_{p=1}^{P} \sum_{\tau=1}^{T}\left(\frac{C_{p \tau}}{C_{\text {tot }}}\right) K_{p \tau} .
$$

With the decompositions defined in (B1)-(B4), the cloud-induced radiation anomaly is given by

$$
\Delta R_{C} \equiv \sum_{p=1}^{P} \sum_{\tau=1}^{T} K_{p \tau} \Delta C_{p \tau}=K_{0} \Delta C_{\text {tot }}+\sum_{p=1}^{P} \sum_{\tau=1}^{T} K_{p \tau}^{\prime} \Delta C_{p \tau}^{*} .
$$

The first term on the rhs is the cloud amount component, which accounts for effects of a change in total cloud cover alone, under the constraint of a fixed distribution of clouds across CTP $-\tau$ categories. This term represents the cloud-induced radiation anomaly that would have resulted from a change in cloud cover obtained by multiplying each cloud fraction appearing in the histogram for the original cloud field by the same factor $\left(=1+\Delta C_{\text {tot }} / C_{\text {tot }}\right)$ and is identical to that derived in Zelinka et al. (2012b).

We can further resolve $K_{p \tau}^{\prime}$ into components:

$$
K_{p \tau}^{\prime}=K_{p}^{\prime}+K_{\tau}^{\prime}+K_{R}^{\prime},
$$

where 


$$
\begin{aligned}
& K_{p}^{\prime}=\sum_{\tau=1}^{T}\left(K_{p \tau}^{\prime} \sum_{p=1}^{P} \frac{C_{p \tau}}{C_{\mathrm{tot}}}\right), \\
& K_{\tau}^{\prime}=\sum_{p=1}^{P}\left(K_{p \tau}^{\prime} \sum_{\tau=1}^{T} \frac{C_{p \tau}}{C_{\mathrm{tot}}}\right),
\end{aligned}
$$

and

$$
K_{R}^{\prime}=K_{p \tau}^{\prime}-K_{p}^{\prime}-K_{\tau}^{\prime}
$$

The cloud-induced radiation anomalies are then expressed as

$$
\begin{aligned}
\Delta R_{C}= & K_{0} \Delta C_{\mathrm{tot}}+\sum_{p=1}^{P}\left(K_{p}^{\prime} \sum_{\tau=1}^{T} \Delta C_{p \tau}^{*}\right) \\
& +\sum_{\tau=1}^{T}\left(K_{\tau}^{\prime} \sum_{p=1}^{P} \Delta C_{p \tau}^{*}\right)+\sum_{p=1}^{P} \sum_{\tau=1}^{T} K_{R}^{\prime} \Delta C_{p \tau}^{*} .
\end{aligned}
$$

The terms on the rhs are the cloud amount, altitude, optical depth, and residual components, respectively. The second term accounts for cloud fraction changes summed over all $\tau$ categories, multiplied by a kernel also summed over all $\tau$ categories but weighted by the total cloud cover found at each $\tau$. Thus, this term results from multiplying an effective kernel accounting for systematic variations with CTP by the total change in cloud fraction at each CTP. Similarly, the third term is calculated from an effective radiative kernel that varies with $\tau$, multiplied by the total change in cloud fraction in each $\tau$ category. Note that, if the radiative kernel is independent of $\tau$, it can be shown that each of the last two terms on the rhs of (B10) vanishes and the cloudinduced anomaly depends only on the vertical distribution of clouds and total cloud cover; there are no optical depth or residual components. Similarly, if the radiative kernel is independent of CTP, there are no altitude or residual components. In contrast, the residual term of Zelinka et al. (2012b) may not vanish in either of these degenerate cases.

\section{REFERENCES}

Andrews, T., and P. M. Forster, 2008: $\mathrm{CO}_{2}$ forcing induces semidirect effects with consequences for climate feedback interpretations. Geophys. Res. Lett., 35, L04802, doi:10.1029/ 2007GL032273.

— J. Jregory, P. Forster, and M. Webb, 2012a: Cloud adjustment and its role in $\mathrm{CO}_{2}$ radiative forcing and climate sensitivity: A review. Surv. Geophys., 33, 619-635, doi:10.1007/ s10712-011-9152-0.

, - - M. J. Webb, and K. E. Taylor, 2012b: Forcing, feedbacks and climate sensitivity in CMIP5 coupled atmosphereocean climate models. Geophys. Res. Lett., 39, L09712, doi:10.1029/2012GL051607.
Armour, K. C., C. M. Bitz, and G. H. Roe, 2013: Time-varying climate sensitivity from regional feedbacks. J. Climate, 26, 4518-4534.

Bala, G., K. Caldeira, and R. Nemani, 2010: Fast versus slow response in climate change: Implications for the global hydrological cycle. Climate Dyn., 35, 423-434, doi:10.1007/ s00382-009-0583-y.

Bony, S., and J. L. Dufresne, 2005: Marine boundary layer clouds at the heart of tropical cloud feedback uncertainties in climate models. Geophys. Res. Lett., 32, L20806, doi:10.1029/ 2005 GL023851.

Cao, L., G. Bala, and K. Caldeira, 2012: Climate response to changes in atmospheric carbon dioxide and solar irradiance on the time scale of days to weeks. Environ. Res. Lett., 7, 034015 , doi:10.1088/1748-9326/7/3/034015.

Cess, R. D., 1974: Radiative transfer due to atmospheric water vapor: Global considerations of the earth's energy balance. J. Quant. Spectrosc. Radiat. Transfer, 14, 861-871.

- 1975: Global climate change: An investigation of atmospheric feedback mechanisms. Tellus, 27, 193-198.

Charney, J. G., and Coauthors, 1979: Carbon dioxide and climate: A scientific assessment. National Academy of Sciences Rep., 22 pp. [Available online at http://www.atmos.ucla.edu/ $\sim$ brianpm/download/charney_report.pdf.]

Collins, W. J., and Coauthors, 2011: Development and evaluation of an Earth-System Model-HadGEM2. Geosci. Model Dev. Discuss., 4, 997-1062.

Colman, R., and B. McAvaney, 2011: On tropospheric adjustment to forcing and climate feedbacks. Climate Dyn., 36,1649-1658, doi:10.1007/s00382-011-1067-4.

_ _ J. Fraser, and L. Rotstayn, 2001: Climate feedbacks in a general circulation model incorporating prognostic clouds. Climate Dyn., 18, 103-122.

Dong, B., J. M. Gregory, and R. T. Sutton, 2009: Understanding land-sea warming contrast in response to increasing greenhouse gases. Part I: Transient adjustment. J. Climate, 22, 30793097.

Doutriaux-Boucher, M., M. J. Webb, J. M. Gregory, and O. Boucher, 2009: Carbon dioxide induced stomatal closure increases radiative forcing via a rapid reduction in low cloud. Geophys. Res. Lett., 36, L02703, doi:10.1029/2008GL036273.

Dufresne, J.-L., and S. Bony, 2008: An assessment of the primary sources of spread of global warming estimates from coupled atmosphere-ocean models. J. Climate, 21, 5135-5144.

Forster, P. M., and K. E. Taylor, 2006: Climate forcings and climate sensitivities diagnosed from coupled climate model integrations. J. Climate, 19, 6181-6194.

Fu, Q., and K. N. Liou, 1993: Parameterization of the solar radiative properties of cirrus clouds. J. Atmos. Sci., 50, 2008-2025.

Garay, M. J., S. P. de Szoeke, and C. M. Moroney, 2008: Comparison of marine stratocumulus cloud top heights in the southeastern Pacific retrieved from satellites with coincident ship-based observations. J. Geophys. Res., 113, D18204, doi:10.1029/2008JD009975.

Gent, P. R., and Coauthors, 2011: The Community Climate System Model version 4. J. Climate, 24, 4973-4991.

Good, P., J. Gregory, and J. A. Lowe, 2011: A step-response simple climate model to reconstruct and interpret AOGCM projections. Geophys. Res. Lett., 38, L01703, doi:10.1029/ 2010 GL045208.

,,,--- and T. Andrews, 2012: Abrupt $\mathrm{CO}_{2}$ experiments as tools for predicting and understanding CMIP5 representative concentration pathway projections. Climate Dyn., 40, 1041-1053, doi:10.1007/s00382-012-1410-4. 
Gregory, J., and M. Webb, 2008: Tropospheric adjustment induces a cloud component in $\mathrm{CO}_{2}$ forcing. J. Climate, 21, 58-71.

— forcing and climate sensitivity. Geophys. Res. Lett., 31, L03205, doi:10.1029/2003GL018747.

Kamae, Y., and M. Watanabe, 2012: On the robustness of tropospheric adjustment in CMIP5 models. Geophys. Res. Lett., 39, L23808, doi:10.1029/2012GL054275.

— , and — 2013: Tropospheric adjustment to increasing $\mathrm{CO}_{2}$ : Its timescale and the role of land-sea contrast. Climate Dyn., doi:10.1007/s00382-012-1555-1, in press.

Klein, S. A., and D. L. Hartmann, 1993: The seasonal cycle of low stratiform clouds. J. Climate, 6, 1587-1606.

— clouds simulated by the ECMWF model. Mon. Wea. Rev., 127, 2514-2531.

—, Y. Zhang, M. D. Zelinka, R. N. Pincus, J. Boyle, and P. J. Gleckler, 2013: Are climate model simulations of clouds improving? An evaluation using the ISCCP simulator. J. Geophys. Res. Atmos., 118, 1329-1342, doi:10.1002/jgrd.50141.

Lu, J., C. Deser, and T. Reichler, 2009: Cause of the widening of the tropical belt since 1958. Geophys. Res. Lett., 36, L03803, doi:10.1029/2008GL036076.

Mace, G. G., S. Houser, S. Benson, S. A. Klein, and Q. Min, 2011: Critical evaluation of the ISCCP simulator using groundbased remote sensing data. J. Climate, 24, 1598-1612.

Marchand, R., T. Ackerman, M. Smyth, and W. B. Rossow, 2010: A review of cloud top height and optical depth histograms from MISR, ISCCP, and MODIS. J. Geophys. Res., 115, D16206, doi:10.1029/2009JD013422.

Meehl, G. A., and Coauthors, 2007: Global climate projections. Climate Change 2007: The Physical Science Basis, S. Solomon et al., Eds., Cambridge University Press, 747-846.

Rossow, W. B., and R. A. Schiffer, 1999: Advances in understanding clouds from ISCCP. Bull. Amer. Meteor. Soc., 80, 2261-2287.

Schneider, S. H., 1972: Cloudiness as a global climatic feedback mechanism: The effects on radiation balance and surface temperature of variations in cloudiness. J. Atmos. Sci., 29, 1413-1422.

_ , and R. E. Dickinson, 1974: Climate modeling. Rev. Geophys., 12, 447-493.

Senior, C., and J. Mitchell, 1993: Carbon dioxide and climate: The impact of cloud parameterization. J. Climate, 6, 393-418.

Shell, K. M., J. T. Kiehl, and C. A. Shields, 2008: Using the radiative kernel technique to calculate climate feedbacks in NCAR's Community Atmospheric Model. J. Climate, 21, 2269-2282.

Sherwood, S. C., W. Ingram, Y. Tsushima, M. Satoh, M. Roberts, P. L. Vidale, and P. A. O'Gorman, 2010: Relative humidity changes in a warmer climate. J. Geophys. Res., 115, D09104, doi:10.1029/2009JD012585.

Soden, B. J., and I. M. Held, 2006: An assessment of climate feedbacks in coupled ocean-atmosphere models. J. Climate, 19, 3354-3360.

_ A. J. Broccoli, and R. S. Hemler, 2004: On the use of cloud forcing to estimate cloud feedback. J. Climate, 17, 3661-3665. , I. M. Held, R. Colman, K. M. Shell, J. T. Kiehl, and C. A. Shields, 2008: Quantifying climate feedbacks using radiative kernels. J. Climate, 21, 3504-3520.
Stephens, G. L., 2010: Is there a missing low-cloud feedback in current climate models? GEWEX News, No. 20, International GEWEX Project Office, Southampton, United Kingdom, 5-7.

Stevens, B., and Coauthors, 2013: The atmospheric component of the MPI-M Earth System Model: ECHAM6. J. Adv. Model. Earth Syst., doi:10.1002/jame.20015, in press.

Taylor, K. E., R. J. Stouffer, and G. A. Meehl, 2012: An overview of CMIP5 and the experiment design. Bull. Amer. Meteor. Soc., 93, 485-498.

Tsushima, Y., and Coauthors, 2006: Importance of the mixed-phase cloud distribution in the control climate for assessing the response of clouds to carbon dioxide increase: A multi-model study. Climate Dyn., 27, 113-126.

von Salzen, K., and Coauthors, 2013: The Canadian Fourth Generation Atmospheric Global Climate Model (CanAM4). Part I: Representation of physical processes. Atmos.-Ocean, 51, 104-125.

Watanabe, M., and Coauthors, 2010: Improved climate simulation by MIROC5: Mean states, variability, and climate sensitivity. J. Climate, 23, 6312-6335.

—, H. Shiogama, M. Yoshimori, T. Ogura, T. Yokohata, H. Okamoto, S. Emori, and M. Kimoto, 2012: Fast and slow timescales in the tropical low-cloud response to increasing $\mathrm{CO}_{2}$ in two climate models. Climate Dyn., 39, 1627-1641, doi:10.1007/s00382-011-1178-y.

Webb, M., C. Senior, S. Bony, and J. J. Morcrette, 2001: Combining ERBE and ISCCP data to assess clouds in the Hadley Centre, ECMWF and LMD atmospheric climate models. Climate Dyn., 17, 905-922.

_, F. Lambert, and J. Gregory, 2013: Origins of differences in climate sensitivity, forcing and feedback in climate models. Climate Dyn., 40, 677-707, doi:10.1007/s00382-012-1336-x.

Wetherald, R., and S. Manabe, 1988: Cloud feedback processes in a general circulation model. J. Atmos. Sci., 45, 1397-1415.

Wyant, M. C., C. S. Bretherton, and P. N. Blossey, 2009: Subtropical low cloud response to a warmer climate in a superparameterized climate model: Part I. Regime sorting and physical mechanisms. J. Adv. Model. Earth Syst., 1, 7, doi:10.3894/ JAMES.2009.1.7.

,,--- , and M. Khairoutdinov, 2012: Fast cloud adjustment to increasing $\mathrm{CO}_{2}$ in a superparameterized climate model. J. Adv. Model. Earth Syst., 4, M05001, doi:10.1029/ 2011MS000092.

Yukimoto, S., and Coauthors, 2011: Meteorological Research Institute-Earth System Model version 1 (MRI-ESM1): Model description. Meteorological Research Institute Tech. Rep. 64, $96 \mathrm{pp}$.

Zelinka, M. D., and D. L. Hartmann, 2010: Why is longwave cloud feedback positive? J. Geophys. Res., 115, D16117, doi:10.1029/ 2010JD013817.

— S. A. Klein, and D. L. Hartmann, 2012a: Computing and partitioning cloud feedbacks using cloud property histograms. Part I: Cloud radiative kernels. J. Climate, 25, 3736-3754.

, —, and — 2012b: Computing and partitioning cloud feedbacks using cloud property histograms. Part II: Attribution to changes in cloud amount, altitude, and optical depth. J. Climate, 25, 3736-3754.

Zhang, M. H., R. D. Cess, J. J. Hack, and J. T. Kiehl, 1994: Diagnostic study of climate feedback processes in atmospheric GCMs. J. Geophys. Res., 99, 5525-5537. 